\title{
Development and validation of an automated FEM-based design optimization tool for continuum compliant structures
}

\author{
Suat Coemert 1 - Baturay Yalvac $\cdot$ Vincent Bott $\cdot$ Yilun Sun $\cdot$ Tim C. Lueth
}

Received: 25 January 2020 / Accepted: 23 June 2020/Published online: 2 July 2020

(C) The Author(s) 2020

\begin{abstract}
This paper presents a design optimization method for continuum compliant structures. The developed optimization tool enables automated design, analysis and optimization of the compliant structures in a single simulation environment. The associated algorithm used automatically analyses the stress distribution occurring under certain loading and deformation conditions of initial designs defined by the user, adjusts a uniform stress distribution among individual flexure hinges by automated dimensioning and finalizes the design by integrating mechanical stops automatically matched with the kinematic capacity of individual hinges. In order to prove the advantages of the proposed optimization method, validation tests were performed under static and dynamic loading conditions. Results of the experiments showed that, compared to the non-optimized ones, optimized structures with the developed tool exhibit more uniform curvatures which indicate more even stress distribution among the individual hinges; up to $25 \%$ value increase in terms of maximum bearable load and maximum permissible deflection angle; less plastic deformation in case of overloading and up to $100 \%$ increased fatigue life.
\end{abstract}

S. Coemert $(\bowtie) \cdot$ B. Yalvac $\cdot$ V. Bott .

Y. Sun · T. C. Lueth

Institute of Micro Technology and Medical Device

Technology (MIMED), Technical University of Munich,

Boltzmannstr. 15, 85748 Garching, Germany

e-mail: suat.coemert@tum.de
Keywords Design optimization - Compliant mechanisms $\cdot$ Automated tool

\section{Introduction}

\subsection{Background}

Minimally invasive surgery (MIS) has established itself in many surgical applications as a standard procedure due to its minimal access methods through small incisions or natural orifices (Oh et al. 2014). MIS brings along many advantages for patients over open surgery such as shorter hospital stay, reduced postoperative pain, improved cosmesis and decreased morbidity. Nevertheless, the surgeons need to face some challenges, since they need to rely on instruments for surgical tasks and endoscopes for visualization. For most of the cases, especially in laparoscopic surgery, available rigid instrumentation is sufficient to perform the interventions smoothly. However, there is still a substantial need for small and flexible instruments for particular applications where rigid instruments reach their limit in terms of optimal accessibility due to anatomical constraints (Schneider et al. 2013). One example of such an application is the endoscopic frontal sinus surgery, where the rigid instruments cannot reach the pathologies more lateral than lamina papyracea (Conger et al. 2014). Another example is 
the transurethral bladder surgery, where rigid instruments cannot provide retroflexion to reach the tumors around the bladder neck (Herrell et al. 2014). In such situations either open surgery becomes the preferred choice or the acceptance of the pathology is chosen over the risk presented by surgery. Under these circumstances, the development of novel small and flexible instruments is of crucial importance.

Compliant mechanisms have a great potential to address the requirements for prospective flexible surgical instruments. First of all, their monolithic structure facilitates miniaturization by eliminating assembly. Secondly, durability can be improved, since no mechanical wear occurs. Lastly, the omission of the lubrication contributes to the sterilizability. Besides these advantages, there are also certain challenges to consider when designing flexible surgical tools based on compliant mechanisms. On the one hand, sufficient flexibility is needed for dexterous movements within the organs and lumens. This usually requires high range of deflections within small distances from the smallest element of compliant mechanism which are the flexure hinges. Metals are usually choice of material if further miniaturization of the overall size is targeted by taking advantage of small wall thicknesses. On the other hand, metals are usually less resilient compared to polymers which results in limited elastic deformability. The use of a superelastic material such as nitinol can therefore address the flexibility requirement in combination with an efficient design. Nevertheless, flexibility is not the only concern when designing flexible surgical tools. Depending on the surgical task, the structure should present sufficient payload capacity to be able to transmit the forces from the user interface to the end effectors. While reachability function can indirectly be associated with the flexibility feature, payload capacity can indirectly be associated with the structure stiffness which is a contrary feature to the former one. This trade-off needs to be analyzed thoroughly and efficient designs should be provided to adapt it to the needs of surgical applications. While doing that, the durability of the system should be respected as a boundary condition. In other words, the designed structure must ensure a satisfactory lifetime under static and dynamic loading conditions, while showing adequate flexibility for reachability and sufficient stiffness for manipulability.

\subsection{State of the art}

Although the number of commercially available surgical instruments based on continuum compliant mechanisms is limited, many research groups are working on the development of such concepts. In the following paragraphs, an overview about design, modelling and optimization of continuum flexure hinge based structures will be provided.

Kutzer et al. (2011) proposed a continuum dexterous manipulator design for minimally invasive treatment of osteolytic lesions. The bidirectional bending of this design is realized with asymmetrical, identical and equally-spaced notches placed on the longitudinal plane of a cylindrical nitinol tube. Swaney et al. (2017) presented a wrist concept which consists of a series of cut-outs that form multiple serial quasi-rectangular cross section flexure hinges. To achieve tip first bending with the aim of reaching small radii of curvature, the flexures closer to the distal tip were designed with lower thicknesses to distribute a larger amount of strains onto these ones. Another good example for the application of continuum compliant structures to create miniaturized designs is the steerable needle. Gerboni et al. (2017) presented a highly articulated flexure hinge based design with less than $1.5 \mathrm{~mm}$ overall diameter which achieves sufficient curvatures for radio-frequency ablation in the liver. The design of the flexible tip is realized with equally dimensioned flexure hinges generated by laser machining of circular notches on a thin walled nitinol tube. Besides these examples of compliant structures that provide planar bending, there are also continuum design configurations offering multiple degrees of freedom. Coemert et al. (2017a) presented how to realize spatially bendable compliant structures using a conventional machining technology, specifically electrical discharge machining. This is achieved by placing the flexure hinges on two perpendicular planes in a serial way. By taking advantage of additive manufacturing technology, implementation of smart structures becomes possible without being constrained by the manufacturing limits. To exemplify, Krieger et al. (2019) came up with shape memory structures that are automatically designed to take predefined task-specific end poses. To achieve that, flexure hinges are placed along the structure in an irregular way that a moment applied by a pull-wire actuation causes the structure to follow a predefined trajectory. Continuum 
compliant mechanism based designs are not limited to the aforementioned examples, but all related works follow similar approaches.

The work related to the modelling of continuum compliant structures mainly concentrates on kinematic modelling. Most of these methods are based on the Euler-Bernoulli beam theory, which determines the behaviour of a beam under certain assumptions such as small bending deformations and constant cross-sectional area. It assumes that the curvature of the beam is proportional to the moment responsible for bending (Howell et al. 2013). Piecewise constant curvature modelling (CCM) was proposed by Webster and Jones (2010) for simplified modelling of continuum structures' poses using joint angles and lengths. Whereas previously mentioned methods cannot address large deflections, Pseudo Rigid Body Model (PRBM) represents a nonlinear approach to the model compliant mechanisms taking these into account. In this method, compliant elements are represented with two rigid linkages connected by a torsion spring (Howell et al. 2013). This model is useful in predicting tip coordinates, whereas correct representation of the deformation profile is not provided. Apart from that, there are inaccuracies related to the shifting of the rotation pivot point in the reality depending on the deflection amount which is not taken into account in the model. Finite Element Method (FEM) is the most reliable tool for kinematic modelling of compliant mechanisms (Druskin et al. 2014). Particularly under the assumption of large deflections, FEM enables the automation of the calculation processes by numerical solving of differential equations. Here the body is converted into finite, infinitesimally small elements with simple geometries (e.g. tetrahedrons). The physical behaviour of these elements is then analysed with regression functions. The smaller the finite elements are selected the more accurate the model is. Increased computational effort should be taken into account due to increasing number of elements. However, if FEM is used for the design of compliant mechanisms, the nonlinear analysis is particularly needed due to large deformations (Friedrich et al. 2014; Friedrich and Lammering 2015).

In order to obtain efficient compliant mechanisms, structural optimization methods are utilized. According to Schumacher (2005), structural optimization methods are investigated in three branches: topology optimization, shape optimization and dimensioning.
Topology describes the location and arrangement of structural elements (Friedrich 2016). There, the size of the design space, boundary conditions, loads and an optimization goal are defined by the user. The design space is discretized by disc or rod elements and calculated by a linear or nonlinear analysis. The geometry optimization deals with the external shape of components and the dimensioning with wall thicknesses and cross-sections. Often these two problems are treated with the same methods. In the development of compliant mechanisms, topology optimization is often the first step in the development process. In this way one gets an idea of the position and connection of the elastic hinges and can thus generate a first draft of the compliant mechanism. The geometry and geometric parameters of the elastic hinges are still unknown. In the following steps, geometry optimization and dimensioning can be used to determine these. Thanks to the advances in 3-D printing, manufacturability of topology optimized compliant structures has drastically improved, especially for plastics. For metals, additive manufacturing methods still need some improvement in terms of minimum producible feature size to realize compliant topology optimized structures, whereas these structures might remain too challenging for conventional manufacturing. Therefore, through dimensioning optimized compliant structures maintain their potential for metals due to their convenient manufacturability. On the other hand, while topology optimization for the synthesis of compliant mechanisms is already well researched and widely used (Zhu et al. 2020; Zheng et al. 2015), there are only occasional approaches for geometry optimization and dimensioning due to the challenges in modelling. Usually, flexure hinge based devices developed for MIS are optimized on a trial-and-error basis. This means that one has to consider a measure to improve the structural properties and then check whether this is actually effective. After the adaptation of the corresponding simulation model, the so-called virtual twin of the real component, the standard use of simulation programs enables an analysis of the stress distribution and other structural properties for static and dynamic load cases. For the determination of this characteristic information, the simulation programs usually use the finite element method (FEM) (Zhang et al. 2011). However, an automated adjustment of certain component variables during the trial-and-error process is not used. For example, Huang et al. (2018) 
investigated the structural behaviour of a snake-like mechanism depending on the dimensioning of individual joints and tried to achieve a constant curvature. The thickness of the hinges is varied along the structure. However, the thickness change is not based on an algorithm and is constant along the structure.

By taking advantage of additive manufacturing, conventional joint geometries can be evolved into novel joints. As an example presented by $\mathrm{Hu}$ et al. (2019), the helical flexure joints have the advantage that the stresses in the structure are distributed more evenly, thus reducing component fatigue. Krieger et al. (2017) developed an algorithm that enables the automated design of flexure hinge based structures, taking into account empirically measured values such as the number of fatigue cycles and the tensile strength of individual hinges. This information is used to evaluate the optimal geometry for a particular hinge. The user simply specifies the desired structural requirements, which are defined by a mass at the proximal end of the structure and the desired number of fatigue cycle. An algorithm then checks the fatigue and tensile strength for different types of geometry to determine the ideal hinge shape. The monolithic production of the polyamide hinges is then carried out by the selective laser sintering process.

\subsection{Limitations of state of the art}

If the implementation of kinematic systems with the aid of compliant mechanisms is considered, certain challenges have to be overcome. Due to the fact that the kinematics are realized by deformations of a material, material parameters such as modulus of elasticity, Poisson's ratio and yield strength, as well as stresses and strains must be taken into account more intensively. The kinematics of the systems can be described by the already mentioned methods (PRBM, CCM, FEM, etc.). The more the assumptions made correspond to reality, the more accurate is the result of the corresponding method. For example, if stresses and strains are not distributed evenly among the flexure elements of a snake-like flexure hinge based structure in case of a distal loading, a method for determining kinematics under the constant curvature assumption would be unsuitable. The previously mentioned examples show that both the design and the constructive implementation of snake-like structures developed for MIS applications are mostly oriented towards constant parameters. This means that a kind of standardized flexure hinge geometry is used to design a continuum flexible mechanism without considering the corresponding boundary conditions. These results in reduced performance or even earlier malfunction of structures, since some flexural elements are exposed to excessive stresses whereas some are not used to their full capacity.

\subsection{Novel approach}

In principle, the use of compliant mechanisms offers some potentials that are currently not being exploited to the full in the development of flexible instruments for MIS. The monolithic production of flexure hinge based structures makes it possible to vary the shape and dimension of individual hinges without incurring costs. The resulting design freedom is currently not being fully exploited, but can have a major influence on the structural dynamic behaviour of the system. To be more specific, deformations and resulting stress distributions can be analysed under defined conditions (loading, fixing, etc.) and adapted by structural optimization. In particular, the stress distribution in the component and the deformation under load can be adjusted. Since manual input of varying parameters would be very time-consuming and expensive, an automated optimization process would make the process much easier at this point.

Generally, in continuum compliant mechanisms with serially arranged flexure hinges of identical geometry, the stiffness or flexibility of the entire structure is increased to achieve a higher payload or deformation for a specific surgical task. In this case, gain in the payload or deformation results in a loss in the other one. However, it is possible under predefined boundary conditions to influence the stress distribution in the individual component and thus increase the payload without reducing the flexibility of the structure by dimensioning individual flexure hinges. This can be done by evaluating the stresses in the area of the individual hinge and subsequently varying certain geometric parameters such as the thickness, length or shape of the hinge. This work deals with the development and validation of an automated design optimization method of flexure hinge based structures. The algorithm used for design optimization uses the finite element method to calculate the displacements and stresses of the compliant mechanism. 
Subsequently, geometric parameters (in this case the thickness) of the flexure hinges are varied in order to adjust the stress distribution in the component. This process is iterative and leads to a structure with optimized flexure hinge geometries.

As for expected advantages of this work, an automated algorithm would simplify the design process of a compliant mechanism and at the same time positively influence the properties of the structure. A higher payload with the same flexibility of the structure would only be one example that would bring many advantages for minimally invasive surgery. With the help of a more even stress distribution, a longer fatigue life of the structure under dynamic loading conditions can be expected. For the same reason, it is also possible to influence the curvature of the structure under load, thus enabling the validity of constant curvature assumption. This would make the kinematic calculations more practical. The listed advantages above will also be validated by experiments under static and dynamic loading conditions. While doing that it will also be questioned if the simulation results match with the reality. Apart from the advantages related to the structure optimization, another distinguishing advantage for the designer is the fact that design, analysis and optimization can take place within the same environment in an automated way which saves the user time, effort and costs.

\section{Implementation}

Figure 1 shows how the optimization concept works. First, the designer should provide the parameters for the initial geometry. This geometry can virtually be constructed using a solid geometry modelling tool and is needed as input data for the Finite Element Analysis (FEA) of the structure. After the initial FEA, the designer explores how the initial design deforms under loading and examines the corresponding stress distribution among the flexural elements. Using this information, the maximum force, under which the manipulator starts to plastically deform (or causes stresses reaching yield strength of the material) is calculated by an iterative process (where the force at the distal end of the structure is increased piecewise). Knowing the maximum permissible force for the nonoptimized structure, the optimization process starts. The iterative optimization process changes the geometry of the hinges in a way that a homogenous distribution of stress on flexure hinges is achieved. After every iteration, this process provides a new set of geometrical parameters for every flexure hinge. As soon as the geometry fulfils the stopping criterion, the optimization process finishes. This is when the desired uniform stress range is achieved on each element. Following the optimization process, the tool numerically calculates the deflection capacity of each flexure hinge to design the angular stops that constrain their bending amount to avoid any plastic deformation so that the optimized structures can get their final shape. In the following sections, the realization of this concept will be explained in detail. The optimization tool is fully implemented in MATLAB (The MathWorks Inc, Natick, Massachusetts) with the help of different toolboxes. The implemented functions include parametric design of structures, adaptive numerical models for the analysis of generated geometries, the optimization process, kinematic analysis and calculations for the angular stops. In order to generate the solid geometries, SG-Library was used which is a constructive MATLAB toolbox developed by Lueth (2015) for spatial modelling of bodies, joints and gears. SG-Library enables its user to create, analyse and manipulate surface models in an automated and parametrized way. For the finite element analysis, Partial Differential Equation (PDE) Toolbox was utilized. With PDE-Toolbox it is possible to solve and analyse linear static problems. The PDE-Toolbox enables its users to import geometry as mesh or STL data which means the data transfer between SGLibrary and PDE-Toolbox is easy and fast since there is no data incompatibility. Additionally, the user does not need to change environments to create and analyse geometries. The PDE-Toolbox provides users with a simple mesh-generator, which creates meshes with triangular (2D) and tetrahedral (3D) elements. On the other hand, PDE-Toolbox is only able to solve linear partial differential equations. Because of this limitation, only the linear elasticity equations can be solved, and small strains can be analysed. Another limitation is that only linear elastic materials can be analysed. Therefore, every material tested in the scope of this work must be isotropic and linear elastic. Considering this fact, Ti6Al4V Grade 5 was chosen as material for its linear elastic properties and comparably higher elasticity among the metals to achieve more flexibility for compliant structures. 
Fig. 1 The optimization concept of continuum compliant structures

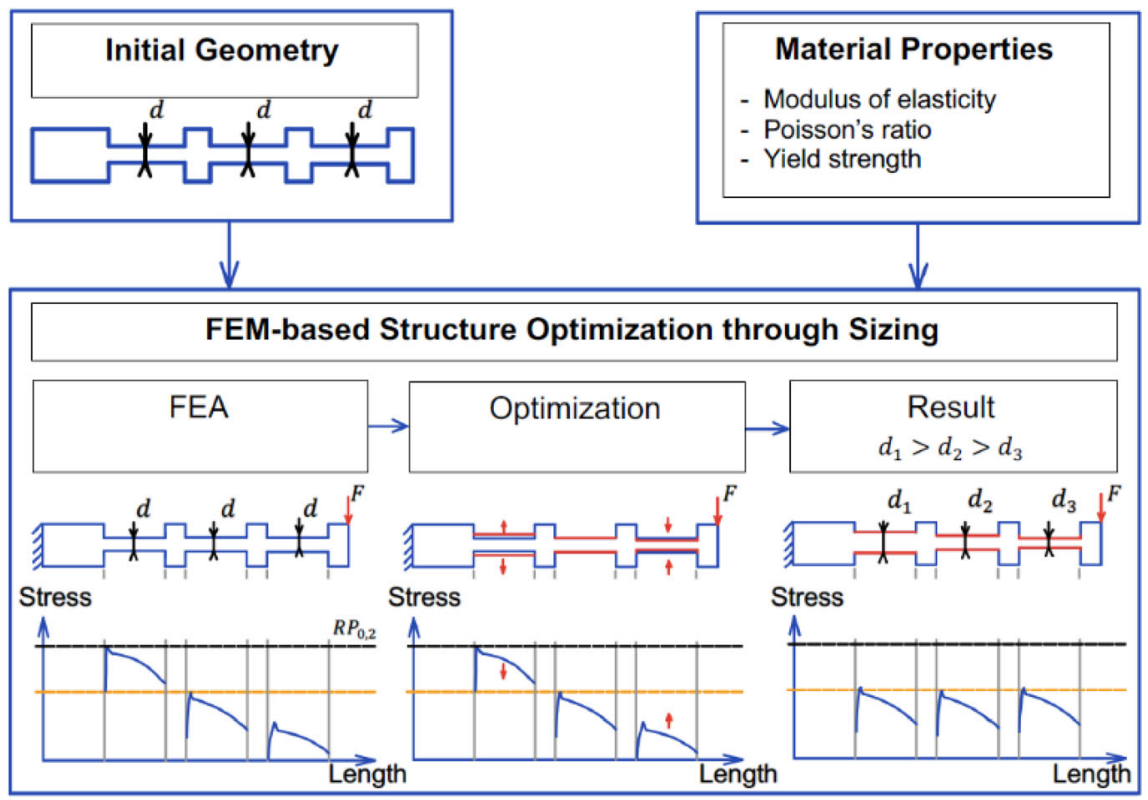

2.1 Input parameters for the initial geometry and FEA

Before performing the first structural analysis, the initial geometry and the material properties need to be defined. Figure 2 shows a representative continuum compliant structure with the geometric input parameters. In Table 1, these geometric parameters are listed with the corresponding nomenclature from the MATLAB script and explained together with the material properties. The two reference stress values sigma_ref_1 $\left(\sigma_{r e f, 1}\right)$ and sigma_ref_2 $\left(\sigma_{r e f, 2}\right)$ listed in the table are each required for different parts of the optimization process:

- sigma_ref_1: For an applied force at the free end of a non-optimized structure, very high stresses
Fig. 2 The initial geometry and the geometric input parameters
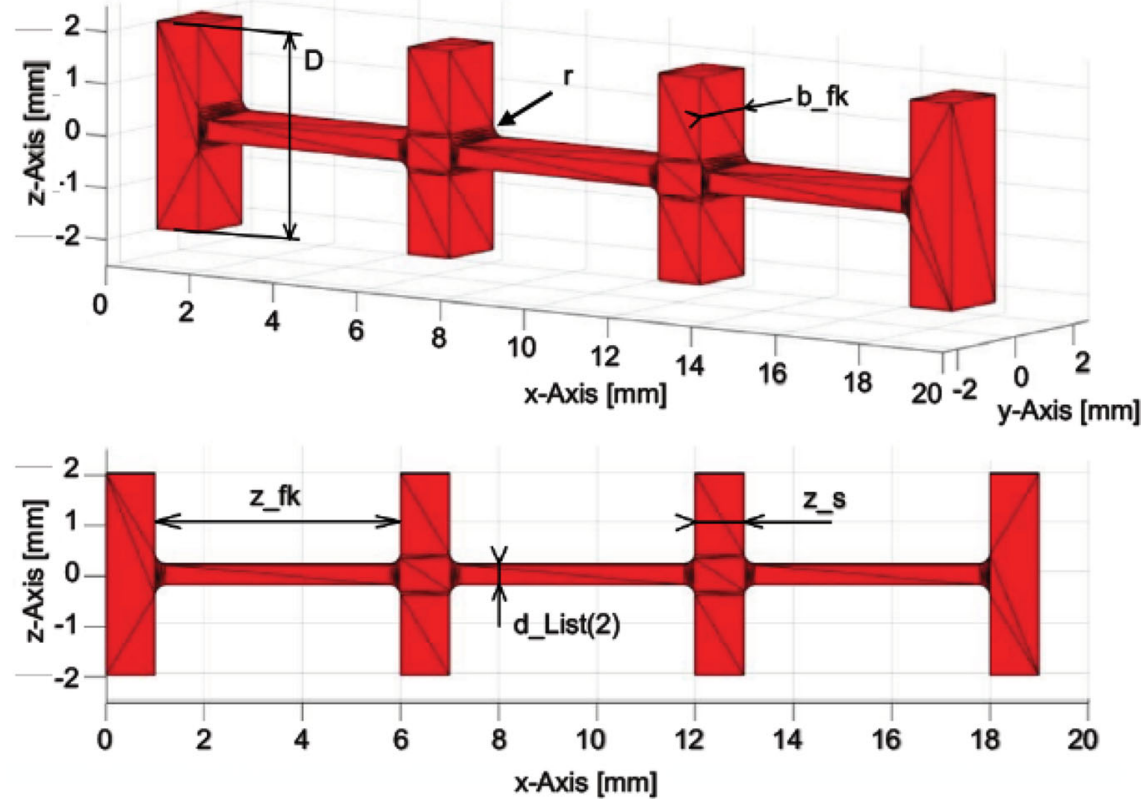
Table 1 All required input parameters before the start of the optimization process

\begin{tabular}{lll}
\hline Parameter & Unit & Definition \\
\hline D & $(\mathrm{mm})$ & Height of the stiff segments \\
d_List (i) & $(\mathrm{mm})$ & Thickness of the respective hinge \\
z_fk & $(\mathrm{mm})$ & Length of each hinge \\
b_fk & $(\mathrm{mm})$ & Width of the compliant structure \\
z_s & $(\mathrm{mm})$ & Length of the stiff segments \\
r & $(\mathrm{mm})$ & Corner radius of hinges \\
gg_max & $(-)$ & Number of rigid segments \\
E_Modul & $(\mathrm{MPa})$ & Elastic modulus $\left(E_{T i}=110\right.$ gpa $)$ \\
PoissR & $(-)$ & Poisson's ratio $\left(v_{T i}=0.33\right)$ \\
RP02 & $(\mathrm{MPa})$ & Yield strength $\left(\sigma_{y i e l d, T i}=948 \mathrm{MPa}\right)$ \\
sigma_ref_1 & $(\mathrm{MPa})$ & First reference stress \\
sigma_ref_2 & $(\mathrm{MPa})$ & Second reference stress \\
\hline
\end{tabular}

occur in the proximal joint, which decreases with increasing distance of the joints from the fixed end. sigma_ref_1 specifies the range at which the stress values for a given load are to be regulated with the help of optimization.

- sigma_ref_2: This defines the maximum allowable stress in the part. This value is relevant for correctly designing the angular stops. If, for example, a high fatigue strength of the structure is desired, a low allowable stress must be selected. However, if a large deflection is relevant, the value should be in the upper stress range. The challenge here is to find a suitable compromise.

\subsection{Working principle of the optimization tool}

In order to ensure the correct understanding of the optimization tool, the individual tasks of each subfunction are described step by step in the following.

\subsubsection{Calculation of the yield force $F_{\max }$}

After the definition of the initial geometry and the material properties by the designer, the first task of the tool is to determine the maximum force the structure can withstand without having any or minimal plastic deformation. Therefore, the simulation model increases the load on the structure in small increments and performs a simulation after every force incrementation. Figure 3 shows the Von Mises equivalent stress (VMS) progression among the flexure hinges during force incrementation. Since the PDEtoolbox is not able to detect plastic deformations, a stop criterion must be determined to stop the simulation and the force incrementation. The stop criterion used for the optimization tool is to compare the maximum VMS occurring in the structure with the yield strength of the material. The simulation series stop, when the toolbox detects, that the maximum equivalent stress among any of the flexure hinges higher than the yield strength of the material. The last applied force is registered as the maximum force $F_{\max }$. In Fig. 4 a visual explanation of this step is provided by means of a flow chart.

\subsubsection{Implementation of the optimization}

Here, the algorithm used targets an even distribution of stress values along the structure by means of dimensioning. To achieve this, the difference $(\Delta \sigma)$ between the initial stress value $\left(\sigma_{V M S}\right)$ of individual hinges and the desired reference stress value $\left(\sigma_{\text {ref }}\right)$ needs to be minimized (see the objective function below). This difference is a function of applied maximum force $F_{\max }$ and geometric dimensions of hinges (length $l$, width $b$, thickness $h$ ) In this work, $F_{\max }$ is always equal to the force leading to the yield stress in the initial (non-optimized) flexure hinge based structures. As other equality constrains, length $l$ and width $b$ were assigned constant values. The thickness $h$ was chosen as the design variable.

$\begin{array}{lll}\text { Min } & \Delta \sigma\left(F_{\text {max }}, l, b, h\right)= & \text { Objective function } \\ & \sigma_{V M S}-\sigma_{\text {ref }} & \\ \text { Subject to } & F_{\text {max }}-F_{\text {yield }, \text { nopt }}=0 & \text { Equality constrains } \\ & l-L=0 & \\ & b-B=0 & \\ & h_{\text {min }} \leq h \leq h_{\max } & \text { Design variable }\end{array}$

The variation of the thickness of individual hinges takes place again in an iterative process. To be more specific, if a flexure hinge has a higher stress level than the (desired) reference stress value $\left(\sigma_{r e f}\right)$, the thickness of the flexure hinge will be increased proportionally to the difference between the measured mean equivalent Von Mises stress $\left(\sigma_{V M S}\right)$ stress along the hinge and the 
Fig. 3 Stress curve of a non-optimized structure after nth iteration. The structure is fixed at $\mathrm{x}=0 \mathrm{~mm}$ and the force acts at $\mathrm{x}=19 \mathrm{~mm}$; the incremental force increase stops when the maximum stress value reaches the yield point $R P_{0.2}$

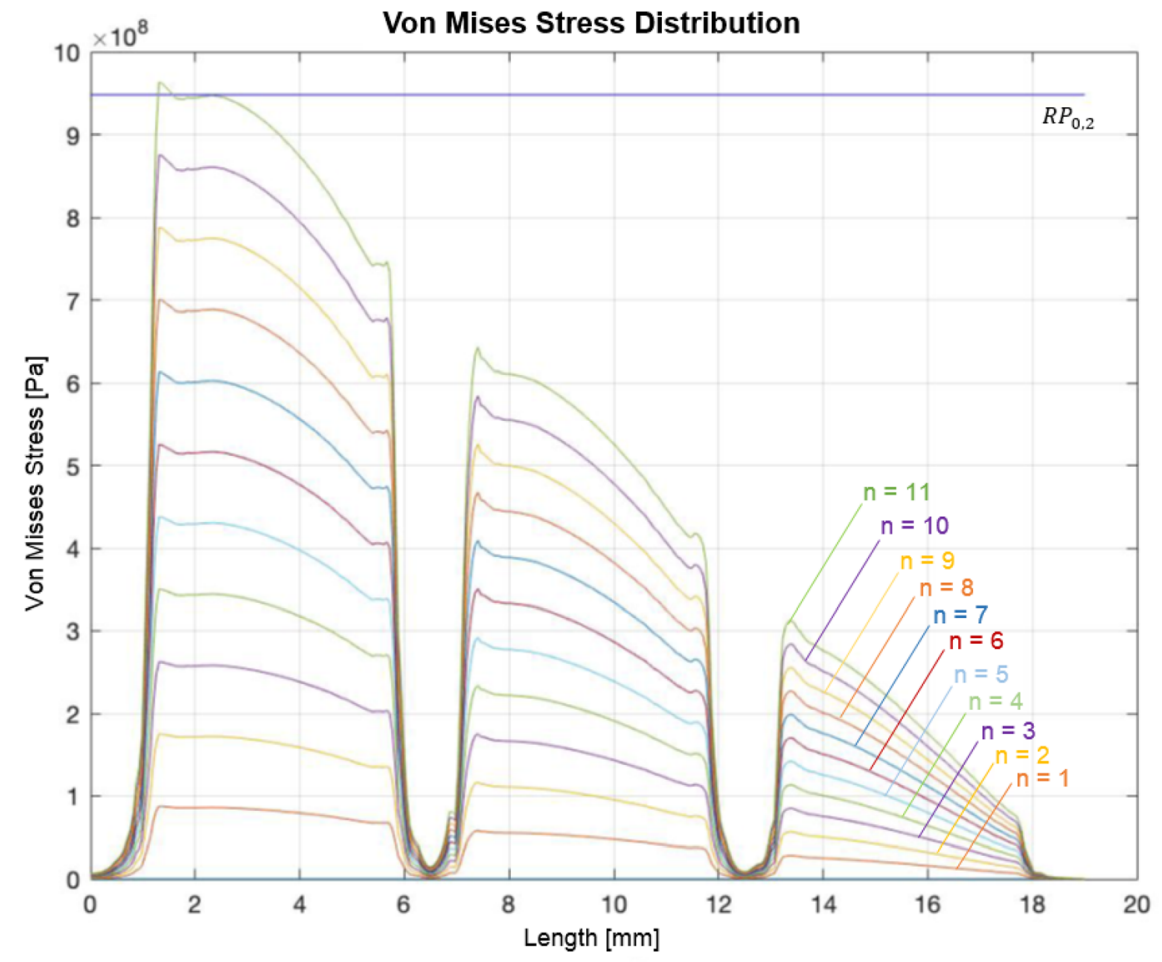

reference stress. The same logic is also applied for the case, in which the measured stress is lower than the reference stress. The thickness of the hinge is decreased in the same way. Following linear equations are implemented in MATLAB for dimensioning of individual hinges:

$k=\frac{0,05}{\sigma_{\text {yield }}} \quad\left[\frac{\mathrm{mm}}{\mathrm{Pa}}\right]$

$\Delta h=k *\left(\sigma_{V M S}-\sigma_{r e f}\right) \quad[\mathrm{mm}]$

$h_{\text {new }}=h_{\text {initial }}+\Delta h \quad[\mathrm{~mm}]$

Here $k$ is the optimization factor. The function of this factor is to transform the stress difference in Pascal into a unit of thickness in mm. By multiplying $k$ with the difference between the calculated stress $\left(\sigma_{V M S}\right)$ and the reference stress $\left(\sigma_{r e f}\right)$, the required thickness change $\Delta h$ can be defined. The optimization is an iterative procedure. After every optimization step, the toolbox starts automatically a new FEA with the new dimensions $\left(h_{\text {new }}\right)$ obtained by adding $\Delta h$ to the previous thickness value $\left(h_{\text {initial }}\right)$ and the maximum force obtained by the first simulation series. The results of the new FEA are used for the next optimization, the new stress profile of the flexure hinges is compared with the stress reference again. The factor is always constant but the difference between the stress on the flexure hinges and the reference value decreases in every step. This iterative process continues until the maximum iteration number, that is defined by the user reached or the difference of the mean equivalent von Mises stress on each flexure hinge is smaller than the defined stresstolerance. The process is explained in the flow chart in Fig. 5.

As one can expect, more accurate results can be achieved through certain modifications such as refining individual iteration steps or reducing tolerances. On the other hand, this conflicts with the required computing time, which also increases with increasing accuracy of the result. It is therefore important to be able to assess whether the chosen settings made lead to an adequate result. For this purpose, post-processing enables a quick assessment by creating all the plots necessary for evaluating the optimization. If one considers the stress distribution of the non-optimized and optimized structure in Fig. 6, it becomes clear that the optimization process was carried out successfully. 


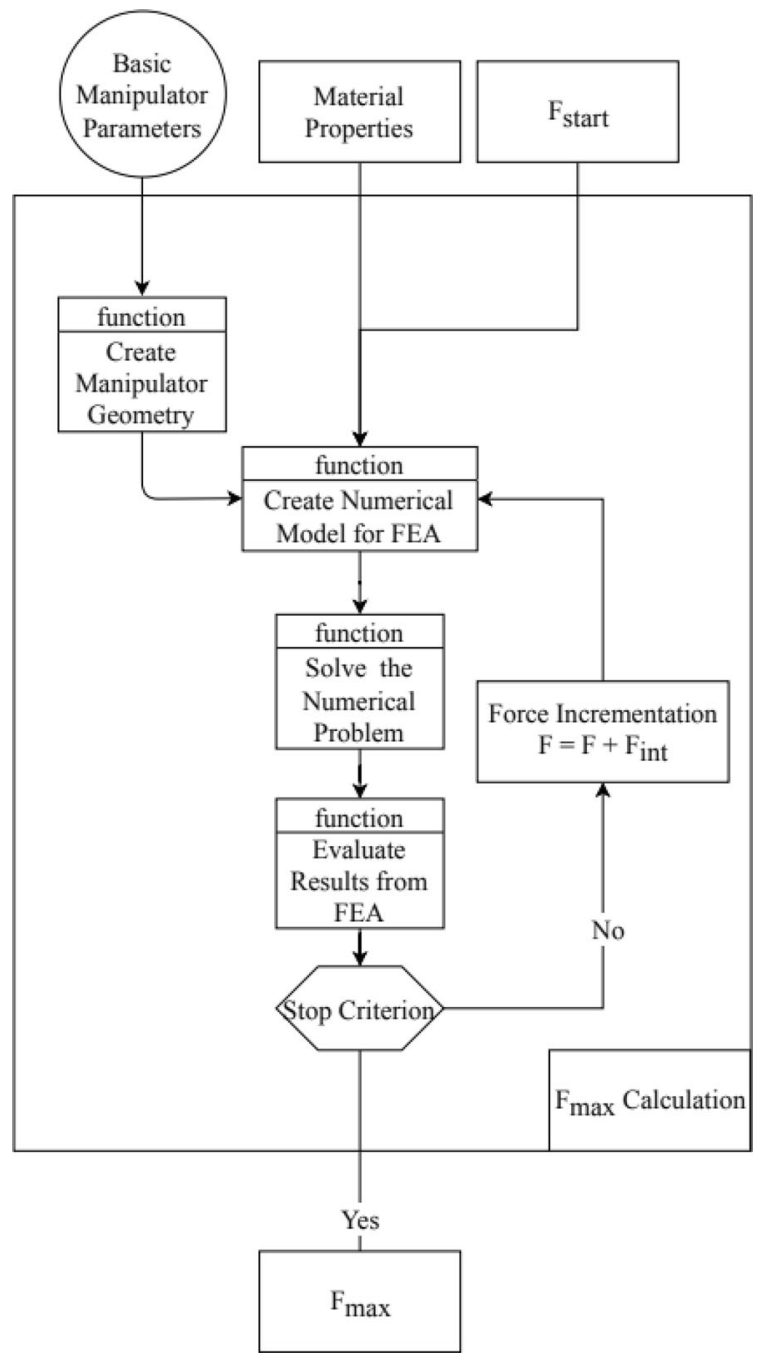

Fig. 4 The algorithm implemented for the calculation of the yield force $F_{\max }$. In this step, a series of numerical problems with the same geometry and boundary conditions are solved. The difference between every simulation is force incrementation. Every simulation has a higher load than the previous one. The simulations and the force incrementations continue until the stop criterion is met

\subsubsection{Adaptation of optimized geometries to kinematic capacity}

In this last step of the optimization process to obtain the final geometry, the user specifies the second reference stress (that can be the yield strength of the material), whereupon the component is loaded in such a way that the maximum occurring stress is again in the range of this value. Tangent lines are then drawn at the distal end of the respective deformed hinge and the angle between the horizontal plane and these tangents is measured. The user receives information about the total deflection of the optimized and non-optimized structure as well as about the deflection of all individual hinges. In addition, the forces applied to generate the deformation are indicated. The results allow an estimation of the stresses occurring under a certain deflection. Moreover, the user can estimate how the total deflection of the structure is distributed among the individual joints. As already mentioned, the angular stops prevent the excessive stress values in the structure. Also, in this case, the second reference stress is used as upper limit. Since the stiffness of each hinge differs after optimization, a calculation must be carried out separately for each of them. Here, the permissible angle is first calculated using the method described above and then an angular stop is added to the structure that prevents this angle from being exceeded. The algorithm of this final optimization step is shown in Fig. 7. Here, it was assumed that the added mass by the integration of angular stops has a negligible influence on the mechanical behavior of the structure.

As shown in Fig. 8 a fully optimized hinge structure looks different than the ones used for analysis in Figs. 2 and 6. Because the angular stops are not needed to constrain the deformation during the analysis stage. Moreover, it can be seen in Fig. 8 that the proximal hinge has higher stiffness with greater thickness. Therefore, the distance between the angular stops is much smaller here than at the distal hinge where the low thickness leads to a higher flexibility.

\subsection{Verification of the solver}

The PDE Toolbox of MATLAB is not commonly used to create and solve finite element models. It has certain limitations, as only linear static analysis can be performed and only linear elastic material models can be used. Therefore, it is necessary to validate the simulations results acquired from PDE Toolbox. To validate the results acquired from PDE-Toolbox, ANSYS 19.2 (ANSYS Inc., Canonsburg, Pennsylvania) was used which is a widely utilized software for linear and non-linear problems of structural mechanics. To increase the significance of the comparison, five different optimized geometries with 4 flexural segments were used for comparative analysis in MATLAB and ANSYS which were varied in 
Fig. 5 The optimization algorithm based on dimensioning

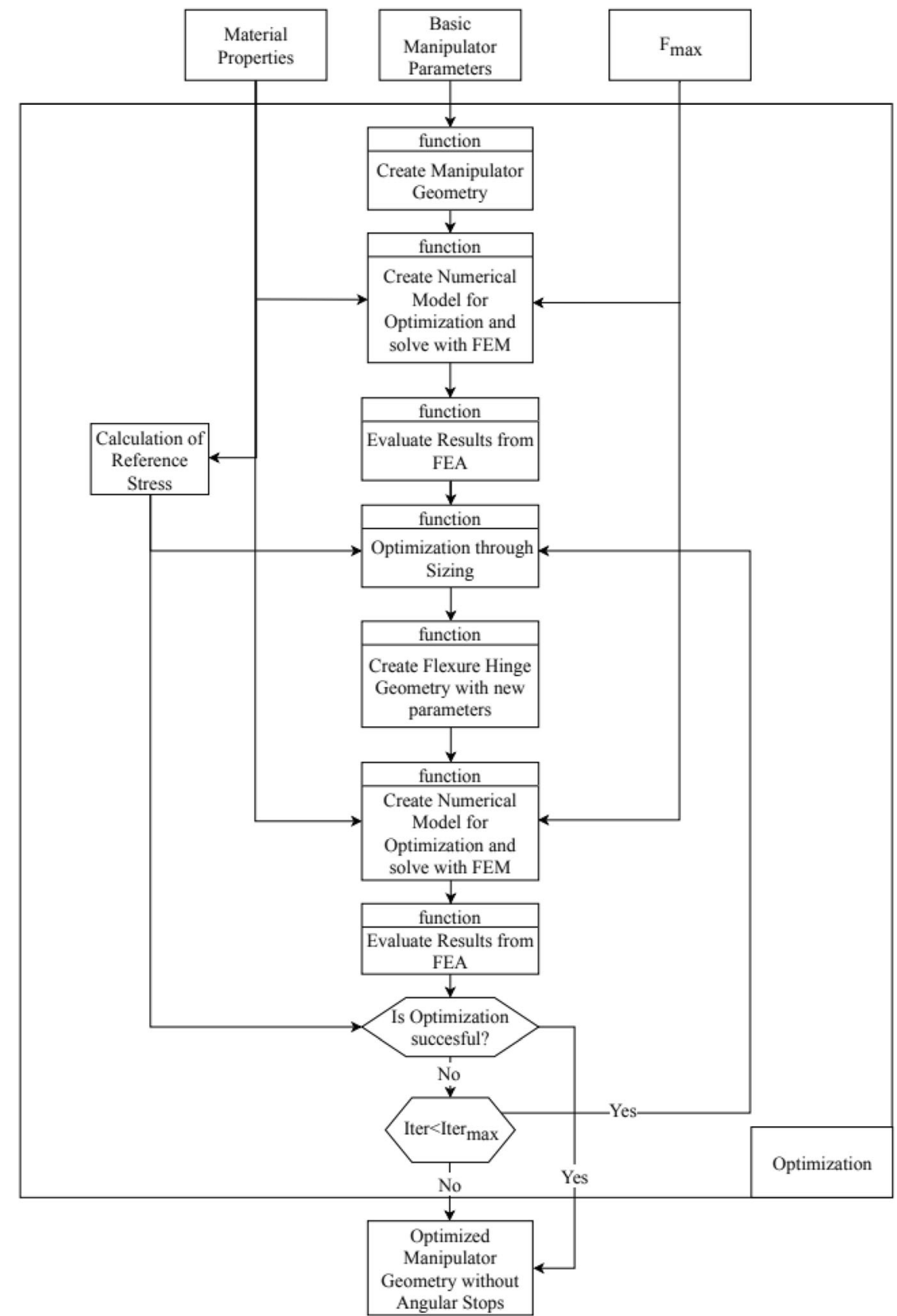

thickness while keeping the length constant and in length while keeping the thickness constant. Three of the samples with $0.3 \mathrm{~mm}$ initial flexure thickness and $3 \mathrm{~mm}, 5 \mathrm{~mm}$ and $7 \mathrm{~mm}$ length. The other two samples had a constant flexure length of $5 \mathrm{~mm}, 0.25$ and 0.35 initial flexure thickness. As material properties, elastic modulus of $110 \mathrm{GPa}$, Poisson's ratio of 0.33 and yield strength of $948 \mathrm{MPa}$ were assigned to the geometries.
In PDE-Toolbox there is no solver setting or manual mesh adjustment. To save computational time and lower the error, the mesh is manually refined on the flexure hinges. A coarser mesh is applied in the angular stops and bulky areas. Therefore, the thickness of the flexure hinges is discretized with at least 3 elements. In ANSYS 19.2 simulation setup, the directsolver is chosen as the solver. The "large deflection" 
Fig. 6 The stress curve of the non-optimized structure (left) is compared to the stress curve of the optimized structure (right); the lower diagrams show the equivalent stresses in the three hinges from (left) and to (right) of the structure optimization



option is activated, and other settings are controlled by the program itself. In Fig. 9 a representative geometry used for the comparative analysis and the meshing differences can be seen. ANSYS uses a stronger meshcreator, which allows the user to concentrate on important areas. On the other hand, PDE-Toolbox does not have any limitations for the maximum number of elements or nodes.

After pre-processing, both geometries are loaded with the same force $F_{\max }$ that is acquired from the toolbox. To compare the results a path was drawn between the proximal and distal point of each flexure hinge. Every path had 50 measurement point. The vonMises-Stress (VMS) value was read on every point on each flexure hinge. In Fig. 10, the comparison of the stress profiles for the most compliant geometry with $0.25 \mathrm{~mm}$ thickness is shown, as the deviations reflect the worst case scenario, since the deformations are the largest for this geometry. The stress profiles for this geometry and the other four geometries were found in good agreement.

As the comparison results suggest, the results acquired from PDE-Toolbox deliver reliable results since the specified optimization reference stress is significantly under the yield strength of the chosen material. Since this deformation range does not lead to great deviations resulting from geometric nonlinearities, it can be concluded that developed optimization can analyze the respective structures reliably.

In order to show the adequateness of the meshing model, convergence analysis was performed with a single flexure hinge structure. Ten cases with the same geometry and boundary conditions but varying mesh refinement were solved. As seen in Fig. 11, the increase in the number of DOF's leads to the convergence of the model. No singularities are observed.

\section{Validation}

In order to validate that the developed optimization tool provides the expected advantages in reality, two sets of experiments were conducted under static and dynamic loading conditions. Before providing detailed information for both test procedures, the test specimens will be introduced in the following, since identical geometries were used for both experiments. The test samples were fabricated using the geometry with constant and variable parameters shown in Fig. 12. 
Fig. 7 The algorithm to adapt the optimized geometries to their kinematic capacity

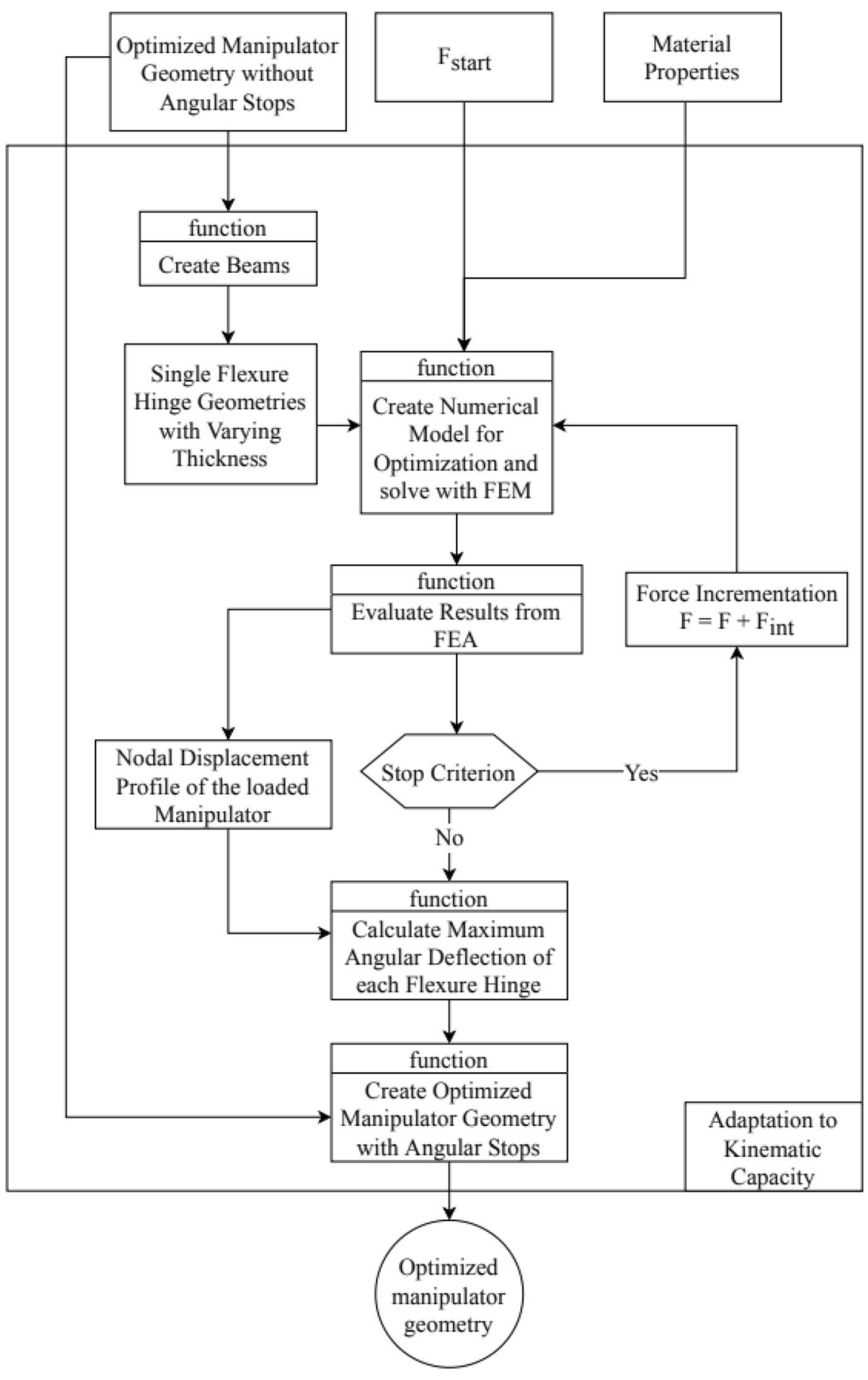

As for material, Ti6Al4V Grade 5 was used due to its high elasticity and homogeneous mechanical properties to obtain reliable results. Due to the very small hinge thicknesses, a precise manufacturing process is required. Therefore the specimens were fabricated with wire electrical discharge machining. In order to ensure that the geometric parameters are within an appropriate tolerance range, the relevant parameters (in particular joint thickness) are measured using a digital caliper with a resolution of $10 \mu \mathrm{m}$. In 
Fig. 8 The final geometry of the optimized compliant structure upon integration of angular stops



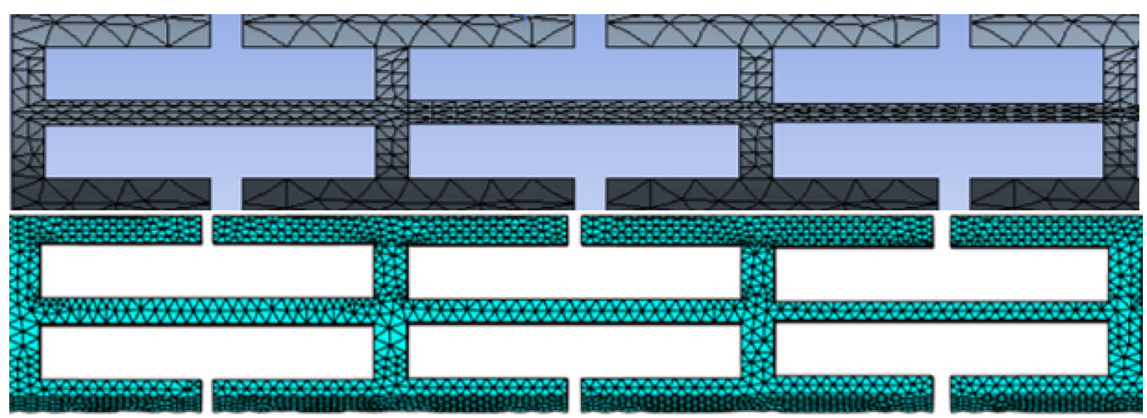

Fig. 9 The representative geometry meshed with ANSYS (top), with PDE-Toolbox (bottom). ANSYS' internal meshcreator creates an inhomogeneous mesh which allows more detailed discretization of regions of interests. PDE-Toolbox meshes homogeneously, therefore regions of interest cannot be discretized finer than the rest of the structure
Fig. 10 The comparison of the stress profiles obtained through ANSYS (blue) and MATLAB (red) for the most compliant geometry with $0.25 \mathrm{~mm}$ thickness and $5 \mathrm{~mm}$ length. (Color figure online)

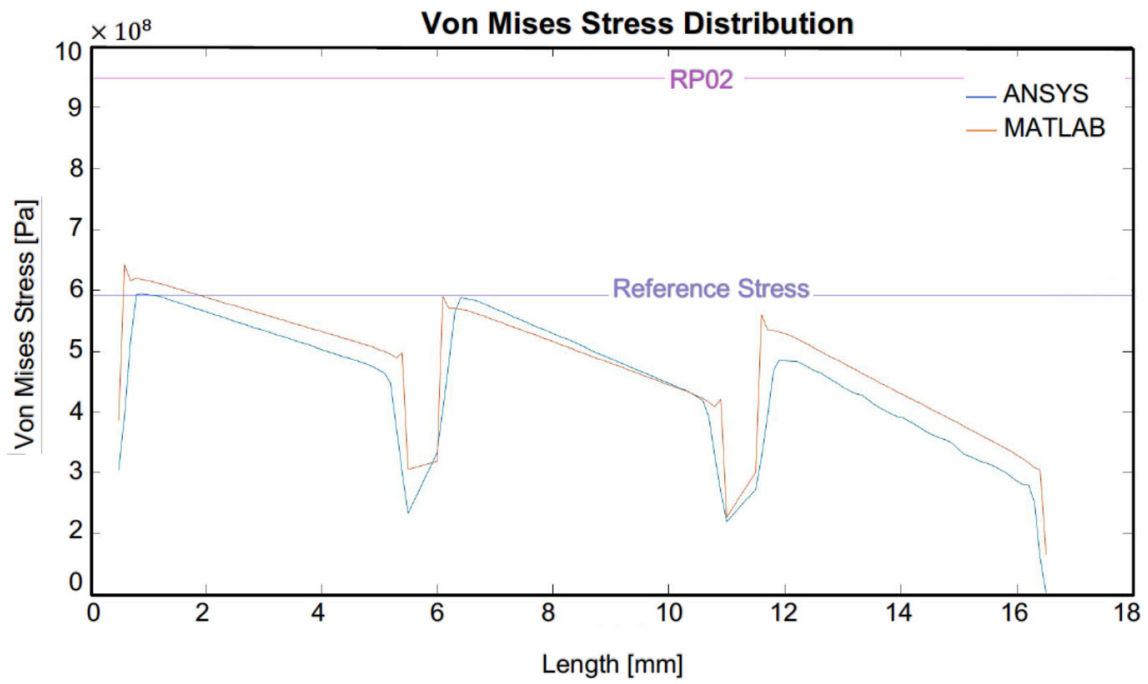

both experiments, the structural behavior of 10 different geometries was tested; 5 of them nonoptimized and 5 of them optimized for comparison purposes. The non-optimized variants differ in the length and thickness of the respective hinges. The optimized geometries differ with varying hinge thickness along the structure, but otherwise resemble the original shape. The varying thicknesses are determined by an iterative process, in which the stresses for a given load case in each hinge are evaluated and 


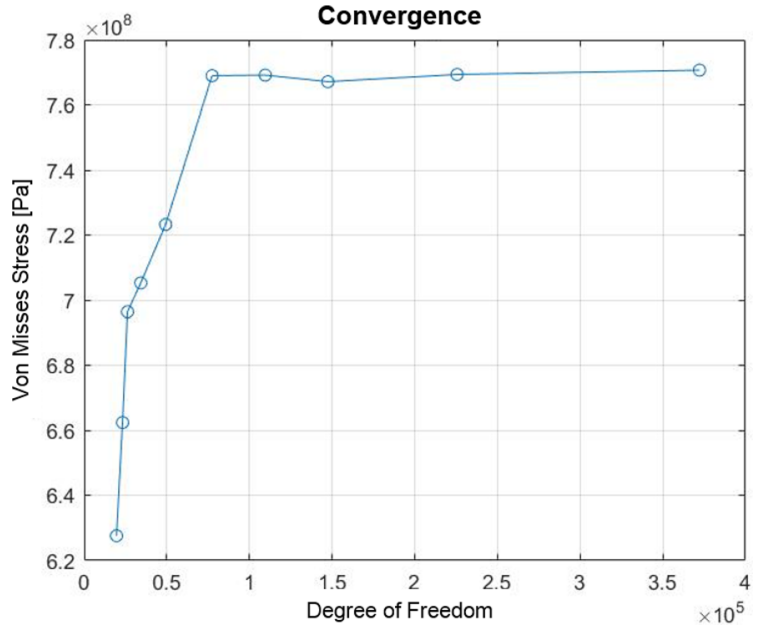

Fig. 11 Convergence analysis to show the adequateness of the meshing model in MATLAB

regulated by structural optimization (variation of hinge thickness), until the stresses in the respective hinges are within a specified tolerance range around the reference stress. All samples are made of a thin Ti6Al4V sheet and therefore have a constant width of $1.7 \mathrm{~mm}$. To provide an overview of the most important parameters, the constant parameters are listed in Table 2 and the varying parameters of the respective variants in Table 3.

The nomenclature of the test specimens is structured in such a way that the constant length of the joints is the first digit. In third place is the information of the original thickness in tenths of a millimeter and finally the information whether the specimen has the optimized or non-optimized hinges (e.g.: 5_0_25_opt). The specimen 5_0_25_opt thus has a hinge length of $5 \mathrm{~mm}$ with an original thickness of $0.25 \mathrm{~mm}$. It is also an optimized joint structure. The values of the hinge thicknesses for the optimized structures in Table 3 result for a reference stress of
Table 2 Constant parameters of test specimens

\begin{tabular}{llcl}
\hline Parameter & Definition & Value & Unit \\
\hline$l_{\text {total }}$ & Total structure length & 33 & $(\mathrm{~mm})$ \\
$D$ & Total structure height & 4 & $(\mathrm{~mm})$ \\
$l_{\text {stiff }}$ & Stiff segment length & 1 & $(\mathrm{~mm})$ \\
$l_{\text {modular }}$ & Modular plug-in length & 5 & $(\mathrm{~mm})$ \\
$l_{\text {stop }}$ & Angular stop length & 1 & $(\mathrm{~mm})$ \\
\hline
\end{tabular}

$\sigma_{r e f, 1}=650 \mathrm{MPa}$. This means that a calculated maximum force for the non-optimized structure causes stresses in the yield strength range $\left(\sigma_{\text {yield }, T i}=948 \mathrm{MPa}\right)$. However, due to a more uniform distribution, the same force only leads to maximum stress values in the optimized structure, which according to the simulation are around $650 \mathrm{MPa}$. Due to the manufacturing tolerances, these values deviate by up to $10 \%$ from the values calculated in the simulation.

\subsection{Validation through static loading tests}

The FEM-based simulation performs linear static calculations in its sub-functions. This means that non-linear effects of large deformations are neglected and plastic deformation is not considered. Therefore, the question arises whether the calculations from the simulation correspond to reality and thus actually contribute to an optimized structure. Therefore, the real forces, angles and thus indirectly also the stresses in the flexure hinge structures should be compared with the values from the simulation. In addition, it will be determined how the optimized and non-optimized structures behave in the plastic region under the same deflection.
Fig. 12 The specimen is fixed on the right side. On the left, a modular plug-in part is attached to which the weights are later attached in order to generate a force in the vertical direction

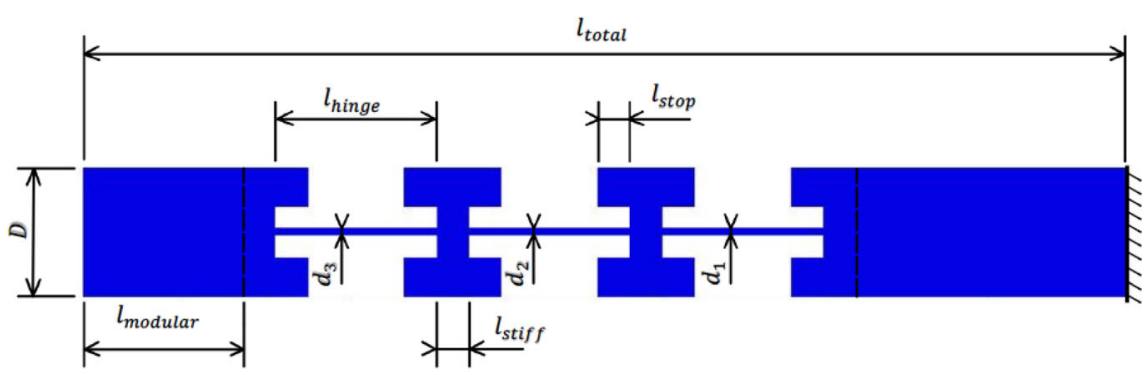


Table 3 Varying parameters of test specimens

\begin{tabular}{llllc}
\hline Specimen & Hinge length $(\mathrm{mm})$ & \multicolumn{3}{l}{ Hinge thickness (measured) $(\mathrm{mm})$} \\
\cline { 4 - 5 } & l hinge & $d_{1}$ & $d_{2}$ & $d_{3}$ \\
\hline 3_0_2_non_opt & 3 & 0.21 & 0.21 & 0.21 \\
3_0_2_opt & 3 & 0.25 & 0.21 & 0.16 \\
4_0_2_non_opt & 4 & 0.21 & 0.21 & 0.21 \\
4_0_2_opt & 4 & 0.23 & 0.19 & 0.16 \\
5_0_2_non_opt & 5 & 0.21 & 0.21 & 0.21 \\
5_0_2_opt & 5 & 0.26 & 0.21 & 0.16 \\
5_0_25_non_opt & 5 & 0.26 & 0.26 & 0.26 \\
5_0_25_opt & 5 & 0.33 & 0.27 & 0.20 \\
5_0_3_non_opt & 5 & 0.31 & 0.31 & 0.31 \\
5_0_3_opt & 5 & 0.37 & 0.32 & 0.22 \\
\hline
\end{tabular}

\subsubsection{Hypothesis}

Beside a good agreement of simulation results with the experimental results, the following findings are expected from static tests to validate the efficiency of the developed tool:

- For the loading values leading to the reference stress value $\sigma_{r e f, 1}=650 \mathrm{MPa}$ in the optimized structure, individual hinges of the optimized structures will exhibit more uniform curvatures compared to the non-optimized ones indicating that the stresses are more evenly distributed along the optimized structures.

- For the loading values leading to the yield stress value $\sigma_{r e f, 2}=948 \mathrm{MPa}$ in both structures, the optimized structures will exhibit larger deflection angles compared to the non-optimized ones withstanding higher amount of forces.

- For the loading values exceeding the yield stress value $\sigma_{\text {ref,2 }}>948 \mathrm{MPa}$, the optimized structures will exhibit significantly lower plastic deformation compared to the non-optimized ones under the exposure of same forces and similar total deflection angles.

\subsubsection{Experimental setup}

The measurement equipment consists of a digital microscope with 9 megapixel resolution (Conrad Electronic SE, Germany) connected to a computer via a USB cable. It is aligned by means of a fixture and does not change its position during the entire experiment. The image is captured using the image processing software ImageJ (NIH, Bethesda, Maryland). The specimen is placed in a specially designed and additively manufactured holder and secured with a screw at the side. A modular plug-in connection allows weights of different masses to be attached from the distal end of the flexure hinge structure. A squared paper in the background allows a rough estimation of the instant angular deflection. Figure 13 shows the most important components of the experimental setup.

\subsubsection{Execution and evaluation of the experiment}

For the execution of the experiment, the load at the distal end is gradually increased, so that a weight leads to a greater bending of the structure. An image is captured after each step and then assigned to the applied force. This procedure is repeated with the optimized hinge structure until the total deflection of the structure is slightly larger than the deformation which corresponds to the calculated value for a stress of $948 \mathrm{MPa}$ from the simulation. Once the maximum load is reached and an image has been generated, the weights are removed and a final image in the unloaded state is generated (Fig. 14). For the non-optimized specimens, the procedure is then repeated, with the graduation of the weights and the maximum load matching that of the optimized specimens. The next step is to determine the deflection angles of individual hinges as well as the total angular deflection of the structure in the image processing software ImageJ. This software includes a tool for measuring the angle between a horizontal line and an additional line which 
Fig. 13 Experimental setup for static experiments: the computer (1), the digital microscope (2), the camera fixture (3), the specimen holder (4), the modular plug-in connection (5), weights (6) and the squared paper (7)



Fig. 14 Series of images showing deformation of a test specimen under increasing load, the last picture corresponds to the load-free fall after removal of the weights (showing plastic deformation)

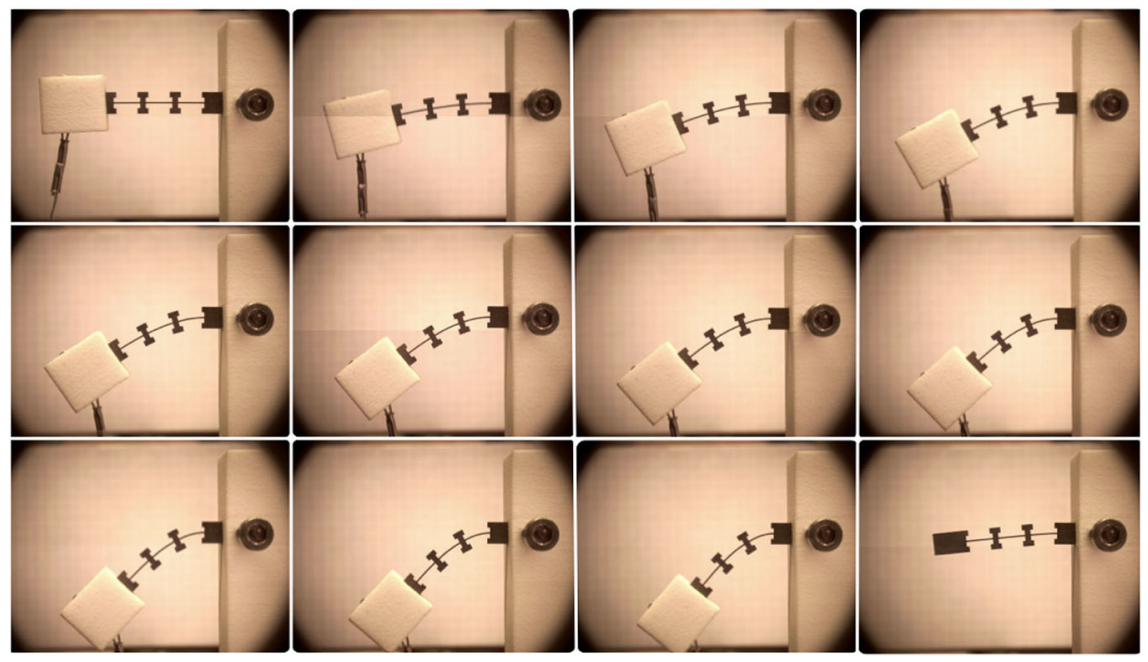

is manually set by the user. The angles are measured as depicted in Fig. 15. Due to the fact that this procedure enables a relatively exact measurement, the maximum deviation between measured and actual angular deflection is anticipated to be less than $0.5^{\circ}$ for most cases. This deviation can be traced back to the manual placement of the lines to measure the angles and would not affect interpretation of the results significantly, as it would always correspond to less than $10 \%$ of the measured angle.

The evaluation provides information about how the total angular deflection is distributed over the three hinges of the sample and thus allows conclusions concerning the curvature along the structure. Furthermore, the total deflection angle can be compared for the optimized and non-optimized structures.
Fig. 15 Procedure for angle measurement in ImageJ
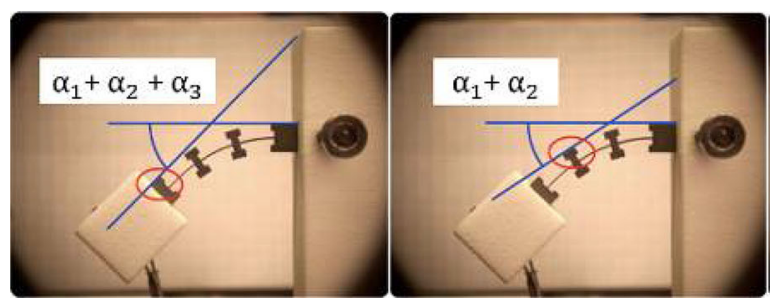
After collecting the data, the information is used to compare the measured values with the values from the simulation and to compare the structural behavior of the optimized and non-optimized specimens. To be more specific, the following information is analyzed in more detail and used to determine comparability between the optimized and the non-optimized structures:

- Individual deflection angles of the hinges $\left(\alpha_{1}, \alpha_{2}, \alpha_{3}\right)$ resulting from total deflection angles $\left(\alpha_{\text {total }}\right)$ which correspond to the defined reference stress value $\sigma_{\text {ref }, 1}=650 \mathrm{MPa}$ in the optimized structure,

- Total yield angles $\left(\alpha_{\text {yield,nopt }}, \alpha_{\text {yield,opt }}\right)$ and respective loads $\left(F_{\text {yield,nopt }}, F_{\text {yield,opt }}\right)$ causing yield stress $\sigma_{r e f, 2}=948 \mathrm{MPa}$ in the optimized and non-optimized structures,

- Total irreversible deflection angles $\left(\alpha_{p s, n o p t}, \alpha_{p s, o p t}\right)$ upon unloading as a plastic deformation measure consequent to the exposure of forces $\left(F_{\text {overload }}\right)$ exceeding the yield stress value $\sigma_{\text {ref }, 2}>948 \mathrm{MPa}$.

\subsection{Validation through dynamic loading tests}

In the next set of experiments, fatigue tests will be used to determine how the structures behave under a dynamic load. In particular, it will be checked whether the optimized specimens have a longer durability than the non-optimized specimens. If this assumption is confirmed, this also induces the applicability of the design optimization for dynamic load cases.

\subsubsection{Hypothesis}

For internal stresses of $650 \mathrm{MPa} \pm 40 \mathrm{MPa}$, the fatigue life for Ti6Al4V is between 100.000 and 250.000 cycles in a strain-controlled fatigue test (Carrion et al. 2017). If this stress range is exceeded and stresses of over $700 \mathrm{MPa}$ are obtained, the number of load changes is reduced to less than 50.000. The dynamic experiments were designed in a way that similar fatigue behavior can be obtained from the specimens. Furthermore, the main expectation from this experiment was that the optimized test specimens can withstand more load cycles than the non-optimized ones under the same dynamic loading conditions.

\subsubsection{Experimental setup}

The experimental setup is shown in Fig. 16. An electric motor is connected to a power source to deflect the test specimens. The rotational movement of the electric motor is converted into a translational movement by means of an eccentric disc and a movable beam. At the end of the beam, steel cables are attached, which are guided via a pulley to the modular plug-in adapter of the test specimens. A further steel cable connects the plug-in adapter with the weights. A fixture is used for the constructive implementation of the fixation boundary condition from the simulation. The plug connection is secured with set screws which eliminate any play. The number of revolutions of the electric motor is achieved by means of a light barrier, which is interrupted once per revolution on the motor. The revolutions are documented by means of a microcontroller BeagleBone. As soon as a sample breaks, the corresponding weight falls on a microswitch. The number of revolutions is stored individually for each sample. Setting the initial angles is performed with the digital microscope and a computer with corresponding ImageJ software which were also used in static experiments.

\subsubsection{Execution and evaluation of the experiment}

For the investigation of the dynamic structural behavior, identical test specimens were used as in the static tests. Just as in the static experiments, ten different geometries were tested here. There were two identical specimens of each geometry. Both the constant and the varying dimensions can be recalled from Tables 2 and 3. In addition to the geometric parameters, the maximum deflection angle of the respective structure variants is also varied. Table 4 gives an overview of the angle and the loading type. Figure 17 illustrates the difference between pulsating and alternating dynamic loading. If the structure is subjected to a pulsating loading, the deflection takes place only in one direction (downwards). In the case of alternating dynamic loading, the structure is first deflected in both directions. The first test was performed with the two variants 4_0_2_non_opt and 4_0_2_opt. As mentioned before, an alternating dynamic loading was applied here. The reason, why the alternating loading case was substituted by pulsating loading will be explained in the discussion 
Fig. 16 Experimental setup for dynamic experiments: power source (1), the electric motor (2), the movable beam (3), the steel cables (4-7), the pulley (5), the modular plug-in connector (6), the weights (8), the microcontroller BeagleBone (9), the light barrier (10) and the microswitch (11)

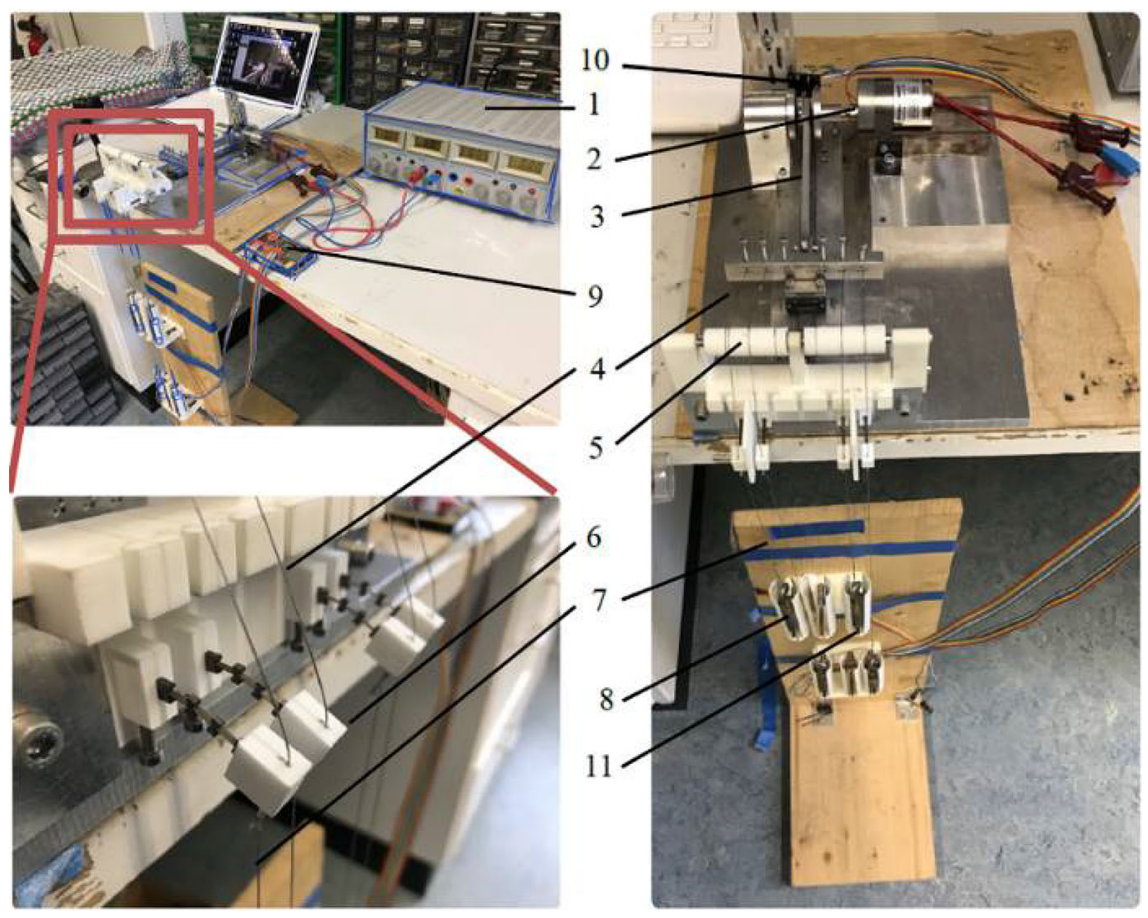

Table 4 Deflection angle and loading type for the dynamic tests

\begin{tabular}{lll}
\hline Specimen geometry (opt. and non-opt.) & Max. deflection angle & Loading type \\
\hline $3 \_0 \_2$ & $\alpha_{d y n \_m a x, 1}=18^{\circ}$ & Pulsating \\
$4 \_0 \_2$ & $\alpha_{d y n \_m a x, 2}=22^{\circ}$ & Alternating \\
$5 \_0 \_2,5 \_0 \_25,5 \_0 \_3$ & $\alpha_{d y n \_m a x, 3}=25^{\circ}$ & Pulsating \\
\hline
\end{tabular}

of the results. In order to ensure the direct comparability of different (initial) thicknesses with a hinge length of $5 \mathrm{~mm}$ were deflected to the same extent. The periodic load was applied at a frequency of $1.5 \mathrm{~Hz}$. During the entire experiment, the mass $m_{d y n}=101.9 \mathrm{~g}$ $\left(F_{\text {dyn_max }}=1 N\right)$ remained the same for the deflection of the specimens. During the tests, only the number of load cycles $N$ up to fatigue fracture was counted. This information is sufficient to clarify the questions formulated at the beginning and to prove the hypothesis.

Before starting the actual tests, it must first be checked which deflection allows a reasonable number of load cycles. The permissible stresses for optimized specimens should therefore be in the range of $650 \mathrm{MPa}$. The stresses of the non-optimized test specimens then result from the deflection that the optimized test specimens show for this stress in the simulation. In order to achieve the desired deflection, the specimens are first clamped. The plug-in adapters are then attached to the corresponding steel cables. The beam, which converts the rotatory movement into a translatory movement, is then positioned in the area of the top dead center. Next, the steel cables are fixed to the beam by means of screws in such a way that the test specimens take the desired angular position $\alpha_{d y n \_ \text {max }, i}$ under load. In order to trigger the microswitch after the fracture of a sample, the orientation of the mass is then adjusted with the aid of set screws. To illustrate the initial condition of the test bench immediately before the test start, the starting position is sketched in Fig. 18. Depending on how the bottom dead center is set via the eccentric disc on the electric 
Fig. 17 The difference between alternating (top) and pulsating (bottom) dynamic loading; $F$ $\left(\alpha_{\text {dyn_max }, i}\right)$ describes the maximum applied force in vertical direction, $\mathrm{N}$ describes a cycle
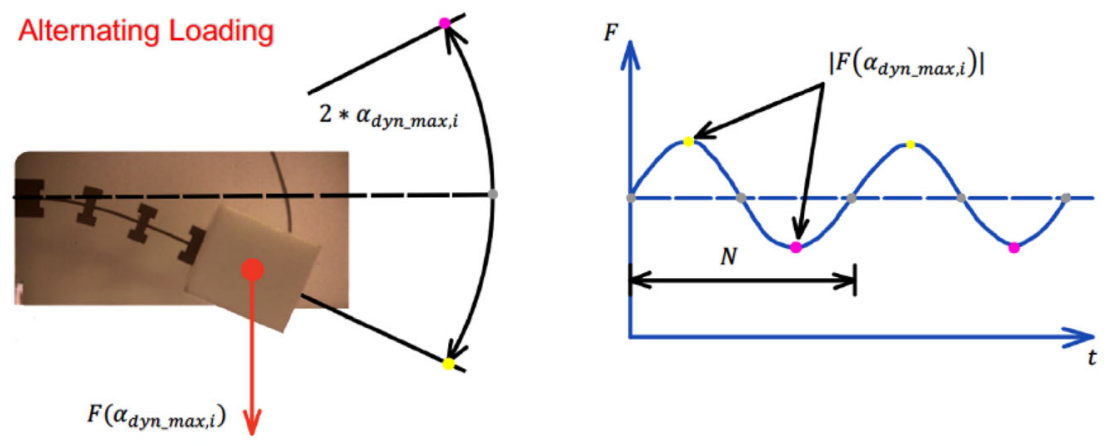

\section{Pulsating Loading}

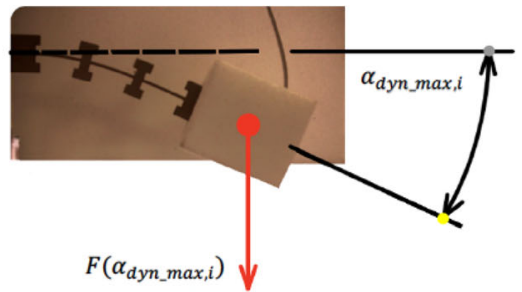

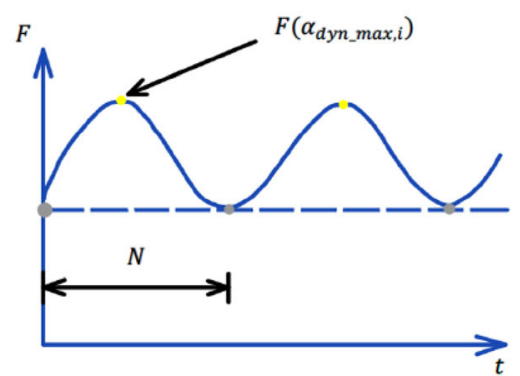

motor, it is possible to adjust whether the loading will be pulsating or alternating.

After the starting position has been set, the test can now be started. To do this, an SD memory card is inserted into the BeagleBone to store the information (number of load cycles) and a current of 15 amperes is set at the power source which corresponds to approximately 90 revolutions per $\min$ or $1.5 \mathrm{~Hz}$ on the
Fig. 18 Starting position of the test bench immediately before the start of the experiment

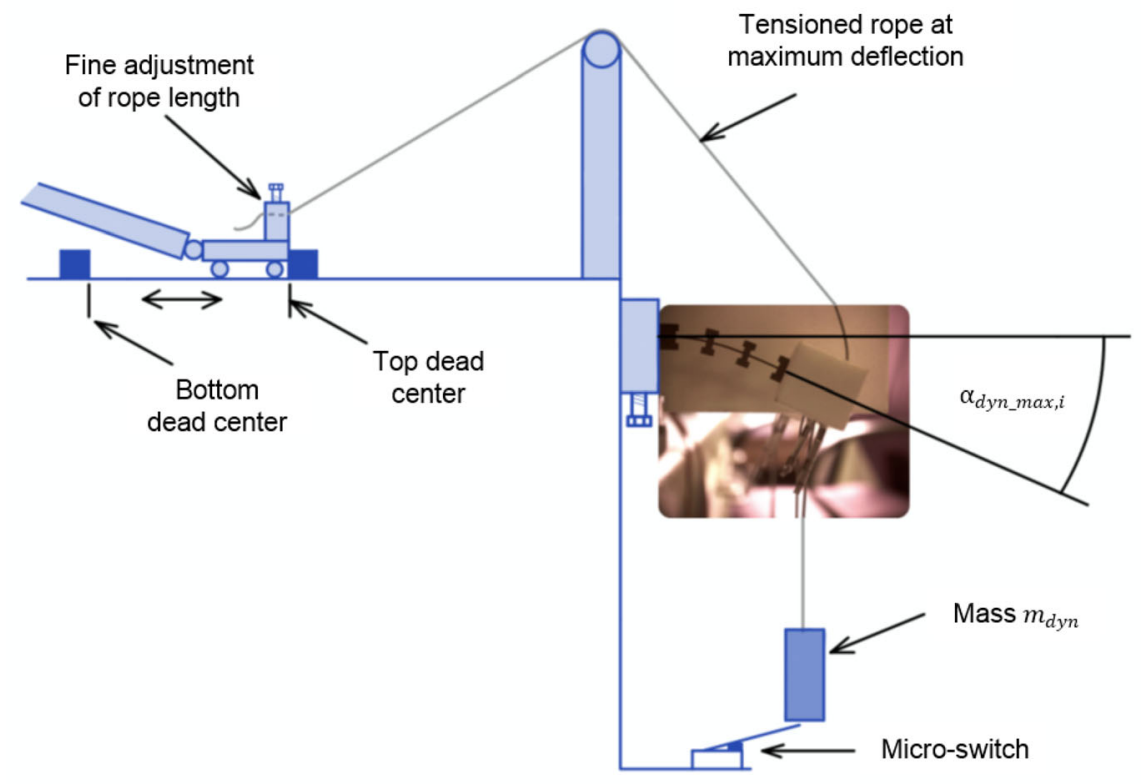


electric motor. After completion of a test run, the number of load cycles can be read from a text file on the SD memory card. This file must be deleted before the next test run.

\section{Results}

\subsection{Results of static loading tests}

Table 5 lists the first series of the measured values; namely the deflection angles of individual hinges $\left(\alpha_{1}, \alpha_{2}, \alpha_{3}\right)$ for all structure variants with a total deflection angle $\left(\alpha_{\text {total }}\right)$ resulting from the simulation for a reference stress of $650 \mathrm{MPa}$ in the respective optimized structure. According to these results, it can be noticed that simulation and experimental results are in good agreement, as the deviation in the deflection angles of individual hinges is in the range of $\pm 0.5^{\circ}$ for about $85 \%$ of the cases. The aforementioned manual measurement accuracy of $\pm 0.5^{\circ}$ might be responsible for certain amount of this deviation. Apart from that, a more uniform distribution of the deflection angles among the individual hinges can be observed. In general, there is a tendency for the non-optimized structure to have a kind of inflexion point at the first hinge indicating an accumulated stress concentration due to having the largest lever arm from the loading point. It can be inferred from the table that the proximal hinge bears up to $55 \%$ of the total deflection angle. On the other hand, a more balanced distribution was observed in the optimized structure, whereas the distal hinge was the most deflected one bearing up to $45 \%$ of the total deflection angle. Figure 19 helps visualizing the difference in the angle distribution between the optimized and the non-optimized samples.

In order to assess further advantages resulting from the optimization, the total deformation angles at the maximum permissible force exposure were analyzed and a comparison between optimized and non-optimized specimens is provided. For this purpose, forces $\left(F_{\text {yield,nopt }}, F_{\text {yield,opt }}\right)$ are calculated in the simulation that lead to a maximum stress of $948 \mathrm{MPa}$ in both structures. This value corresponds to the yield strength of Ti6Al4V. The angular deflections $\left(\alpha_{\text {yield,nopt }}, \alpha_{\text {yield,opt }}\right)$ resulting from these forces during the measurements are then compared between the nonoptimized and optimized structures. The results of these measurements can be found in Table 6. It can be concluded from the results that the optimized specimens can withstand a higher force and at the same time allow a larger maximum deflection. A value increase between 7 and $24 \%$ for the maximum force can be observed, as the stiffness of the structure decreases. Similarly, a value increase between 6 and $19 \%$ for the maximum total deflection angle can be observed.
Table 5 Simulation and experimental results for deformation angles of individual hinges of the optimized and the nonoptimized specimens at a reference stress of $650 \mathrm{MPa}$ in the optimized structures

\begin{tabular}{llccc}
\hline Specimen & Total deflection angle & \multicolumn{2}{c}{ Deflection angle of individual hinges } \\
\cline { 3 - 5 } & $\alpha_{\text {total }}$ & $\alpha_{1}$ & $\alpha_{2}$ & $\alpha_{3}$ \\
\hline 3_0_2_opt (sim) & $28.2^{\circ}$ & 6.8 & 8.6 & 12.8 \\
3_0_2_opt (exp) & & 7.2 & 8.2 & 12.8 \\
3_0_2_non_opt (exp) & & 13.9 & 9.1 & 5.2 \\
4_0_2_opt (sim) & $34.9^{\circ}$ & 9.6 & 11.4 & 13.9 \\
4_0_2_opt (exp) & & 9.9 & 10.9 & 14.1 \\
4_0_2_non_opt (exp) & & 19.4 & 11.1 & 4.4 \\
5_0_2_opt (sim) & $41.1^{\circ}$ & 12.6 & 15.1 & 13.4 \\
5_0_2_opt (exp) & & 13.0 & 14.9 & 13.2 \\
5_0_2_non_opt (exp) & & 23.7 & 13.1 & 4.3 \\
5_0_25_opt (sim) & $33.7^{\circ}$ & 8.3 & 10.7 & 14.7 \\
5_0_25_opt (exp) & & 8.7 & 11.6 & 13.4 \\
5_0_25_non_opt (exp) & & 17.7 & 11.3 & 4.7 \\
5_0_3_opt (sim) & 28.7 & 7.2 & 8.1 & 13.4 \\
5_0_3_opt (exp) & & 7.0 & 8.4 & 13.3 \\
5_0_3_non_opt (exp) & & 13.2 & 10.1 & 5.4 \\
\hline
\end{tabular}




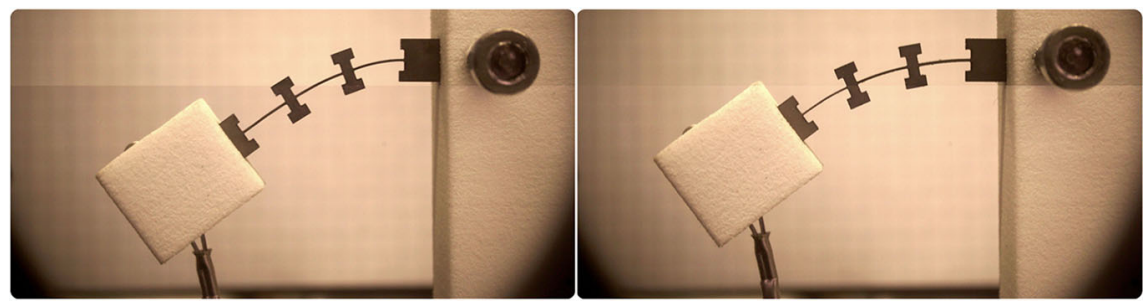

Fig. 19 Comparison of the curvature profile between a non-optimized (left) and an optimized (right) test specimen at a reference stress of $650 \mathrm{MPa}$; specimen left: 5_0_2_non_opt, specimen right: 5_0_2_opt

Table 6 Maximum permissible forces and total deflection angles for the optimized and the nonoptimized specimens

\begin{tabular}{lllll}
\hline Specimen & $F_{\text {yield,nopt }}(\mathrm{N})$ & $F_{\text {yield,opt }}(\mathrm{N})$ & $\alpha_{\text {yield,nopt }}\left({ }^{\circ}\right)$ & $\alpha_{\text {yield,opt }}\left({ }^{\circ}\right)$ \\
\hline 3_0_2 & 0.86 & 1.07 & 32.2 & 36.7 \\
4_0_2 & 0.80 & 0.97 & 38.7 & 46.2 \\
5_0_2 & 0.70 & 0.87 & 43.3 & 45.9 \\
5_0_25 & 1.0 & 1.21 & 36.5 & 40.0 \\
5_0_3 & 1.44 & 1.54 & 36.0 & 39.1 \\
\hline
\end{tabular}

In the last part of this experiment, the plastic deformation occurring in both optimized and nonoptimized structures under the exposure of same forces was investigated. The stress state of the optimized specimens was slightly above the yield point immediately before complete unloading. The concentrated stresses in the non-optimized specimens were higher. This could be confirmed particularly by the permanent deformation of the test specimens after complete unloading (Fig. 20). The permanent deflection angles of all specimens upon unloading are shown in Table 7. The assumption that the optimized structures will exhibit significantly lower plastic deformation compared to the non-optimized ones can be confirmed with these results.

\subsection{Results of dynamic loading tests}

Table 8 shows the number of load cycles for all relevant specimens rounded to thousands. Both the maximum deflection and the load type are also given.
Table 7 Irreversible deflection angles of the optimized and the non-optimized specimens under the exposure of the same forces leading to stresses exceeding yield strength

\begin{tabular}{llll}
\hline Specimen & $F_{\text {overload }}(\mathrm{N})$ & $\alpha_{p s, \text { nopt }}\left({ }^{\circ}\right)$ & $\alpha_{p s, \text { opt }}\left({ }^{\circ}\right)$ \\
\hline 3_0_2 & 1.47 & 6.3 & 2.0 \\
4_0_2 & 1.47 & 4.7 & 2.4 \\
5_0_2 & 1.28 & 4.3 & 1.7 \\
5_0_25 & 1.96 & 5.8 & 0.7 \\
5_0_3 & 2.16 & 3.8 & 0.2 \\
\hline
\end{tabular}

Due to the small number of test specimens, the results are considered individually. There is no calculation of a mean value. Although the amount of data collected is very small, the relevant results of the test specimens subjected to pulsating dynamic loading are consistent and unambiguous. The number of average load cycles of optimized specimens was between 50 and $100 \%$ higher than that of non-optimized specimens. As
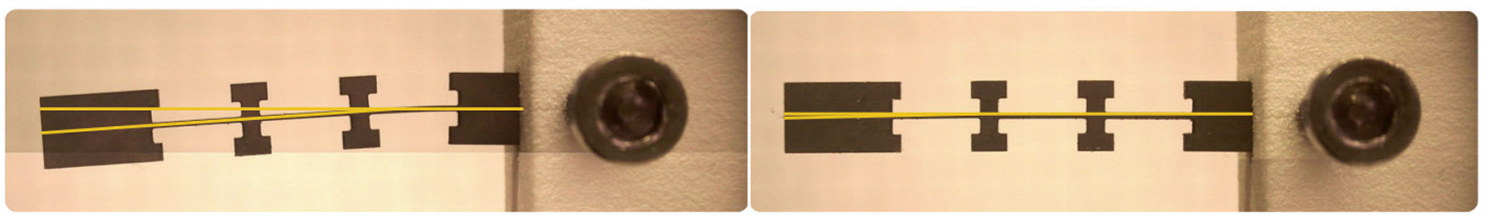

Fig. 20 Plastic deformation after overloading; non-optimized specimen left: 5_0_25_non_opt, optimized specimen right: 5_0_25_opt 
Table 8 Number of achieved load cycles for all test specimens

\begin{tabular}{lllll}
\hline Specimen geometry & $\alpha_{\text {dyn_max }}\left({ }^{\circ}\right)$ & Loading type & \#N $\left(\times 10^{3}\right)$ non_opt & \#N $\left(\times 10^{3}\right)$ opt \\
\hline 3_0_2 (1) & $18^{\circ}$ & Pulsating & 30 & 45 \\
3_0_2 (2) & & & 32 & 47 \\
4_0_2 (1) & $22^{\circ}$ & Alternating & 12 & 8 \\
4_0_2 (2) & & & 13 & 12 \\
5_0_2 (1) & $25^{\circ}$ & Pulsating & 27 & 57 \\
5_0_2 (2) & & & 34 & 67 \\
5_0_25 (1) & & & 28 & 49 \\
5_0_25 (2) & & 34 & 60 \\
5_0_3 (1) & & 23 & 38 \\
5_0_3 (2) & & 26 & 38 \\
\hline
\end{tabular}

already mentioned, the deflection of the test specimens with a hinge length of $5 \mathrm{~mm}$ was performed with a maximum angle of $25^{\circ}$. The bar chart in Fig. 21 shows that the number of load cycles is reduced with increasing (initial) hinge thickness. This was expected since the same amount of deflection causes higher stresses in the stiffer structures which results in a reduced durability.

\section{Discussion}

With the conducted experiments, the following advantages of the developed optimization tool could be validated:
- The developed method results in a more uniform curvature along the structure that indicates more uniform stress distribution along the structure. The bearable loads and the maximum permissible deflection angles increase significantly for the optimized geometries.

- For the loading cases leading to stresses exceeding the yield strength of the material, optimized structures exhibit less plastic deformation than the non-optimized ones.

- The optimization leads to an improved durability with up to $100 \%$ increase in the load cycles for pulsating dynamic loading conditions. This improvement could not be proven for the alternating dynamic loading conditions.

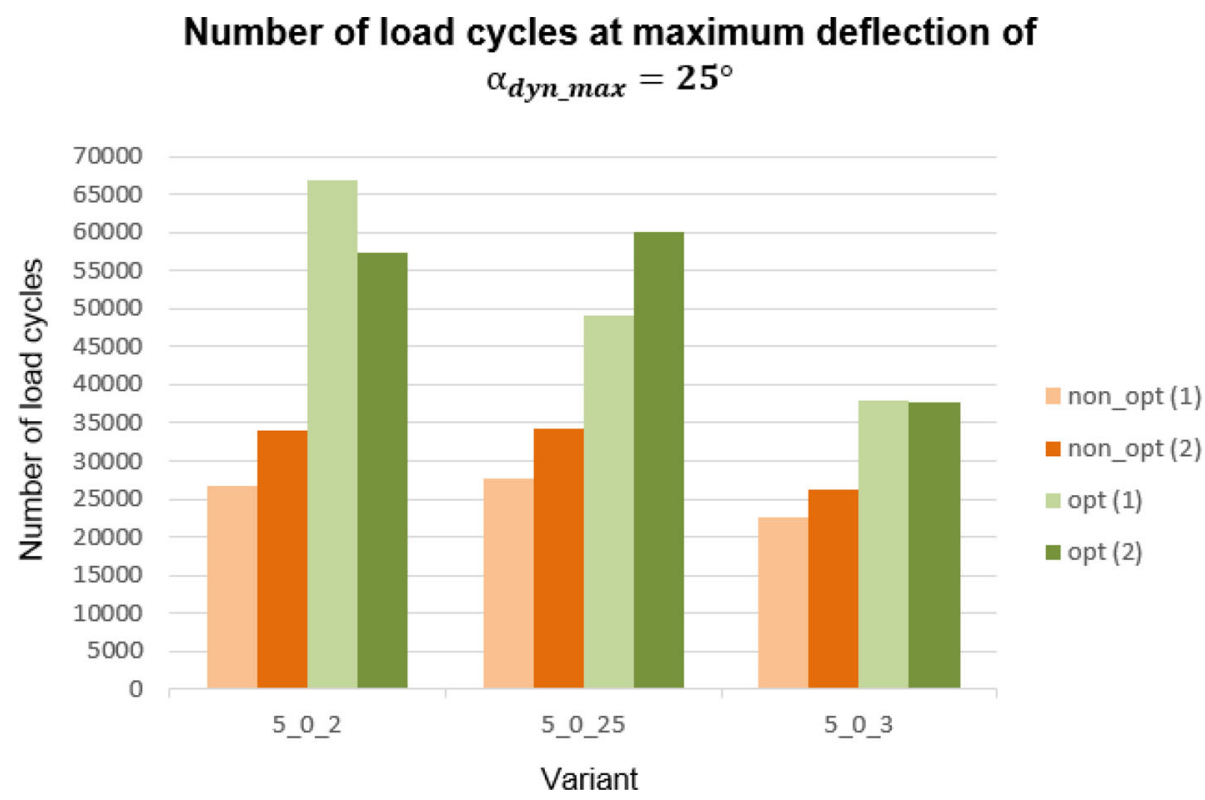

Fig. 21 Comparison of the achieved load cycles for all test specimens with $5 \mathrm{~mm}$ hinge length 
Despite the limitation that only linear static analysis can be performed in PDE-Toolbox, the abovementioned advantages could be validated for relatively larger deflections offered by the resilience of Ti6Al4V. The reliability of the results was also examined by performing comparative analysis in ANSYS. Adequateness of mesh refining in PDEToolbox was verified using convergence analysis.

The optimization tool is also able to perform its algorithms for more complex geometries. However, the geometries demonstrated in this work were kept simple in order to limit the manufacturing complexity and costs to a reasonable level.

Whereas topology optimization is very promising for realizing complex geometries with additive manufacturing methods, the developed optimization method based on dimensioning is particularly useful for miniaturized structures to be manufactured with conventional methods. Additive manufacturing technology is yet not in the desired level to realize miniaturized compliant structures due to the limitations regarding minimum producible feature sizes (wall thickness, minimum hole diameter etc.) (Coemert et al. 2017b).

Besides these advantages, the optimization tool currently has the limitation that it cannot use nonlinear material models. Especially if much larger deflections are expected from snake-like structures with high dexterity requirements, use of non-linear materials such as superelastic nitinol become indispensable. Therefore, future implementation of a solver which enables the use of non-linear materials would be very crucial to increase applicability of the tool.

In the following, the potential underlying causes will be discussed why the constant curvature behavior and improvement in the fatigue behavior for alternating loading could not be validated. In the simulation, the optimization process only takes place for the area of hinge structure. This means that the constructive implementation of the clamping and the exact method of force exposure in the real loading case are not taken into account. In order to reduce the costs of the test specimens in particular, a modular plug-in part was designed, which was later used to fasten the weights. This constructive conversion led to an enlarged lever arm, which increased the moment at the hinges by a not negligible factor compared to the simulation. Figure 22 demonstrates the relevant variables. In the experiments, the influence of the enlarged lever arm mainly affected the deformation of the joints of optimized structures. Particularly in the hinge at the distal end of an optimized specimen relatively large angles could be detected which were not predicted by the simulation. The reason for this observation could have the following causes:

- The lever arm, which in combination with the force causes a moment at the distal hinge, is more than doubled in all specimens for this hinge.

- The hinge at the distal end of an optimized structure is usually the thinnest and therefore more flexible than other hinges. This leads to a particularly high sensitivity to changed moments.

Table 9 shows the angles resulting from the original simulation for the geometry 3_0_2_opt at $650 \mathrm{MPa}$ and compares these with the measured values for the same deflection. In order to check whether the changed load case in the experiments actually led to the deviations of the angles, a further simulation was carried out. Here the load case was adapted to the real conditions. The newly calculated simulation values confirm the assumption that the enlarged lever arm is the cause of for the deviating results in the experiment. In order to achieve a constant curvature, either the load case in the simulation must be adapted to the real boundary conditions or vice versa. Ideally, this would result in a uniform angular distribution for the reference stress defined in the simulation.

If we go back to the results of the dynamic tests and review the results for the alternatingly loaded specimen geometry 4_0_2 in Table 8, it can be seen that the optimized samples failed earlier or around the same cycle with the non-optimized samples. If we look at the configuration of the alternating loading system, it can be assumed that during a cycle undesired forces, moments and stresses occur in the test specimen. The reason for this assumption is the fact that the rope and thus the rope tension is not vertical, but has a variable inclination with respect to the vertical axis. In order to prevent this phenomenon and bring the configuration closer to the simulation conditions, the remaining test specimens were subjected to pulsating loading. As it can clearly be seen in Table 8 , this measure has led to more univocal results. 


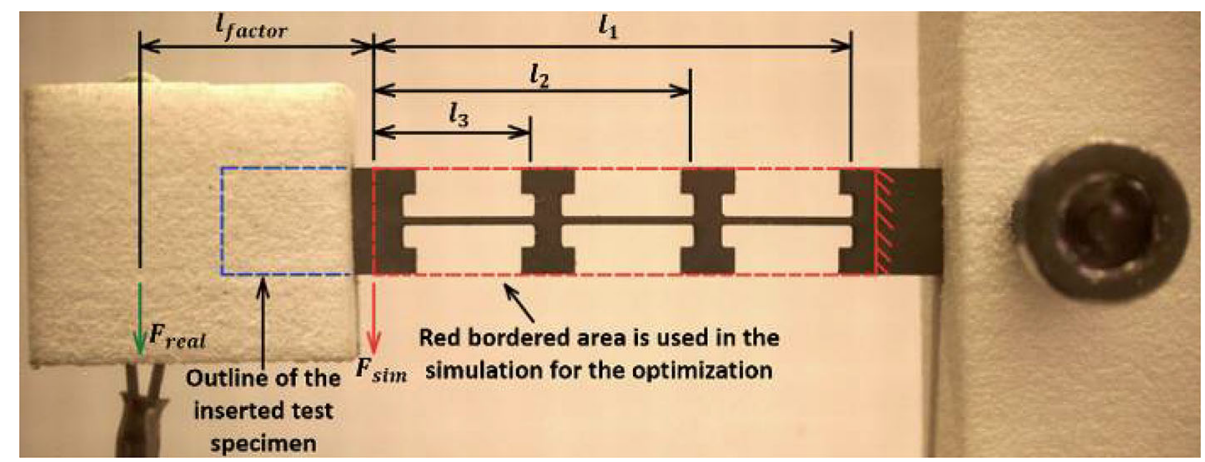

Fig. 22 Influence of the increased lever arm on the measurement results

Table 9 Angular deflections for the specimen 3_0_2_opt; comparison between measurement, initial and adapted simulation

\begin{tabular}{lccccc}
\hline Specimen & $\alpha_{\text {total }}$ & $\alpha_{1}$ & $\alpha_{2}$ & $\alpha_{3}$ & $\mathrm{~F}(\mathrm{~N})$ \\
\hline 3_0_2_opt (exp) & $24.9^{\circ}$ & 6.0 & 6.8 & 12.1 & 0.49 \\
3_0_2_opt (sim_old) & $24.9^{\circ}$ & 8.0 & 8.4 & 8.5 & 0.98 \\
3_0_2_opt (sim_new) & $24.5^{\circ}$ & 6.1 & 6.9 & 11.5 & 0.58 \\
\hline
\end{tabular}

\section{Conclusion}

In order to maximize the benefits of minimally invasive surgery and extend its application to wider spectrums, there is a need for small and strong flexible surgical instruments, since rigid instruments sometimes are too limited in terms of reachability in complicated anatomies. Continuum compliant designs have high potential for such prospective flexible instruments thanks to their monolithic structure enabling miniaturized sterilizable structures. In order for such designs to fulfill the requirements of the surgical intervention in terms of flexibility-stiffness trade-off and durability, efficient designs are essential. Although many research groups propose design solutions based on continuum flexure hinge based structures, design methods lack consideration of how internal stresses are distributed along the structure under certain loading and deflection conditions and resulting negative effects on the system performance and durability. Starting from this, we developed a design optimization tool for continuum flexure hinge based structure, which incorporates automated design, analysis and optimization of structures in a single environment.
The conducted validation experiments proved that the developed optimization tool increases the performance of continuum compliant structures in terms of maximum bearable force and maximum deflection angle. Additionally, more uniform stress and stress distribution and correspondingly increased fatigue life were proven by means of experiments.

For future work, modeling and integration of nonlinear materials such as superelastic nitinol into the developed optimization tool can greatly enhance its applicability, since such materials are the most suitable for such applications due to their high deformability.

Acknowledgements Open Access funding provided by Projekt DEAL.

Open Access This article is licensed under a Creative Commons Attribution 4.0 International License, which permits use, sharing, adaptation, distribution and reproduction in any medium or format, as long as you give appropriate credit to the original author(s) and the source, provide a link to the Creative Commons licence, and indicate if changes were made. The images or other third party material in this article are included in the article's Creative Commons licence, unless indicated otherwise in a credit line to the material. If material is not included in the article's Creative Commons licence and your intended use is not permitted by statutory regulation or exceeds the permitted use, you will need to obtain permission directly from the copyright holder. To view a copy of this licence, visit http://creativecommons.org/licenses/by/4.0/.

\section{References}

Carrion, P.E., Shamsaei, N., Daniewicz, S.R., Moser, R.D.: Fatigue behavior of Ti-6Al-4V ELI including mean stress effects. Int. J. Fatigue 99, 87-100 (2017) 
Coemert, S., Kollmer, M., Olmeda, M., Krieger, Y.S., Brecht, S.V., \& Lueth, T.C.: Development of a double arm endoscopic mini-manipulator system for transurethral resection of bladder tumors (TURBT). In: 2017 IEEE/RSJ International Conference on Intelligent Robots and Systems (IROS), pp. 1670-1676. IEEE, New York (2017a, Sept.)

Coemert, S., Traeger, M.F., Graf, E.C., Lueth, T.C.: Suitability evaluation of various manufacturing technologies for the development of surgical snake-like manipulators from metals based on flexure hinges. In: Procedia CIRP, pp. 1-6 (2017b)

Conger Jr., B.T., Illing, E., Bush, B., Woodworth, B.A.: Management of lateral frontal sinus pathology in the endoscopic era. Otolaryngol. Head Neck Surg. 151(1), 159-163 (2014)

Druskin, V., Simoncini, V., Zaslavsky, M.: Adaptive tangential interpolation in rational Krylov subspaces for MIMO dynamical systems. SIAM J. Matrix Anal. Appl. 35(2), 476-498 (2014)

Friedrich, R.: Modellierung und Optimierung nachgiebiger Mechanismen auf Basis elastischer Festkörpergelenke mit Hilfe von nichtlinearen Finiten Balkenelementen. Ph.D. Thesis. Helmut-Schmidt-Universität (2016)

Friedrich, R., Lammering, R.: Flexure hinge mechanisms modeled by nonlinear Euler-Bernoulli-Beams. PAMM 15(1), 193-194 (2015)

Friedrich, R., Lammering, R., Rösner, M.: On the modeling of flexure hinge mechanisms with finite beam elements of variable cross section. Precis. Eng. 38(4), 915-920 (2014)

Gerboni, G., Greer, J.D., Laeseke, P.F., Hwang, G.L., Okamura, A.M.: Highly articulated robotic needle achieves distributed ablation of liver tissue. IEEE Robot. Autom. Lett. 2(3), 1367-1374 (2017)

Herrell, S.D., Webster, R., Simaan, N.: Future robotic platforms in urologic surgery: recent developments. Curr. Opin. Urol. 24(1), 118 (2014)

Howell, L.L., Magleby, S.P., Olsen, B.M. (eds.): Handbook of Compliant Mechanisms. Wiley, Berlin (2013)

Hu, Y., Zhang, L., Li, W., Yang, G.Z.: Design and fabrication of a 3-D printed metallic flexible joint for snake-like surgical robot. IEEE Robot. Autom. Lett. 4(2), 1557-1563 (2019)

Huang, J., Wang, H., Tian, S., Zhang, F.: Modeling of notched variable stiffness continuum flexible snake-like robot. In: 2018 International Automatic Control Conference (CACS), pp. 1-6. IEEE, New York (2018, Nov.)

Krieger, Y.S., Kuball, C.M., Rumschoettel, D., Dietz, C., Pfeiffer, J.H., Roppenecker, D.B., Lueth, T.C.: Fatigue strength of laser sintered flexure hinge structures for soft robotic applications. In: 2017 IEEE/RSJ International Conference on Intelligent Robots and Systems (IROS), pp. 1230-1235. IEEE, New York (2017, Sept.)
Krieger, Y.S., Schiele, S., Detzel, S., Dietz, C., Lueth, T.C.: Shape memory structures-automated design of monolithic soft robot structures with pre-defined end poses. In: 2019 International Conference on Robotics and Automation (ICRA), pp. 9357-9362. IEEE, New York (2019, May)

Kutzer, M.D., Segreti, S.M., Brown, C.Y., Armand, M., Taylor, R.H., Mears, S.C.: Design of a new cable-driven manipulator with a large open lumen: preliminary applications in the minimally-invasive removal of osteolysis. In: 2011 IEEE International Conference on Robotics and Automation, pp. 2913-2920. IEEE, New York (2011, May)

Lueth, T.C.: SG-Library: Entwicklung einer konstruktiven MATLAB-Toolbox zur räumlichen Modellierung von Körpern, Gelenken und Getrieben. In: 11. Kolloquium Getriebetechnik, pp. 183-203 (2015)

Oh, S.Y., Kwon, S., Lee, K.G., Suh, Y.S., Choe, H.N., Kong, S.H., Lee, H.J., Kim, W.H., Yang, H.K.: Outcomes of minimally invasive surgery for early gastric cancer are comparable with those for open surgery: analysis of 1013 minimally invasive surgeries at a single institution. Surg. Endosc. 28(3), 789-795 (2014)

Schneider, J.S., Burgner, J., Webster III, R.J., Russell III, P.T.: Robotic surgery for the sinuses and skull base: what are the possibilities and what are the obstacles? Curr. Opin. Otolaryngol. Head Neck Surg. 21(1), 11 (2013)

Schumacher, A.: Optimierung Mechanischer Strukturen. Springer, Berlin (2005)

Swaney, P.J., York, P.A., Gilbert, H.B., Burgner-Kahrs, J., Webster, R.J.: Design, fabrication, and testing of a needlesized wrist for surgical instruments. J. Med. Dev. 11(1), 0145011-0145019 (2017)

Webster III, R.J., Jones, B.A.: Design and kinematic modeling of constant curvature continuum robots: a review. Int. J. Robot. Res. 29(13), 1661-1683 (2010)

Zhang, D., Gao, Z., Fassi, I.: Design optimization of a spatial hybrid mechanism for micromanipulation. Int. J. Mech. Mater. Des. 7(1), 55-70 (2011)

Zheng, J., Yang, X., Long, S.: Topology optimization with geometrically non-linear based on the element free Galerkin method. Int. J. Mech. Mater. Des. 11(3), 231-241 (2015)

Zhu, B., Zhang, X., Zhang, H., Liang, J., Zang, H., Li, H., Wang, R.: Design of compliant mechanisms using continuum topology optimization: a review. Mech. Mach. Theory 143, 103622 (2020)

Publisher's Note Springer Nature remains neutral with regard to jurisdictional claims in published maps and institutional affiliations. 